\title{
One Percent Determination of the Primordial Deuterium Abundance*
}

\author{
Ryan J. Cooke ${ }^{1,5}$ (1) Max Pettini ${ }^{2,3}$, and Charles C. Steidel ${ }^{4}$ (1) \\ ${ }^{1}$ Centre for Extragalactic Astronomy, Department of Physics, Durham University, South Road, Durham DH1 3LE, UK; ryan.j.cooke@durham.ac.uk \\ ${ }^{2}$ Institute of Astronomy, Madingley Road, Cambridge CB3 OHA, UK \\ ${ }^{3}$ Kavli Institute for Cosmology, Madingley Road, Cambridge CB3 OHA, UK \\ ${ }^{4}$ California Institute of Technology, MS 249-17, Pasadena, CA 91125, USA \\ Received 2017 July 26; revised 2018 January 26; accepted 2018 January 27; published 2018 March 12
}

\begin{abstract}
We report a reanalysis of a near-pristine absorption system, located at a redshift $z_{\text {abs }}=2.52564$ toward the quasar Q1243+307, based on the combination of archival and new data obtained with the HIRES echelle spectrograph on the Keck telescope. This absorption system, which has an oxygen abundance $[\mathrm{O} / \mathrm{H}]=-2.769 \pm 0.028(\simeq 1 / 600$ of the solar abundance), is among the lowest metallicity systems currently known where a precise measurement of the deuterium abundance is afforded. Our detailed analysis of this system concludes, on the basis of eight D I absorption lines, that the deuterium abundance of this gas cloud is $\log _{10}(\mathrm{D} / \mathrm{H})=-4.622 \pm 0.015$, which is in very good agreement with the results previously reported by Kirkman et al., but with an improvement on the precision of this single measurement by a factor of $\sim 3.5$. Combining this new estimate with our previous sample of six high precision and homogeneously analyzed $\mathrm{D} / \mathrm{H}$ measurements, we deduce that the primordial deuterium abundance is $\log _{10}(\mathrm{D} / \mathrm{H})_{\mathrm{P}}=-4.5974 \pm 0.0052$ or, expressed as a linear quantity, $10^{5}(\mathrm{D} / \mathrm{H})_{\mathrm{P}}=2.527 \pm 0.030$; this value corresponds to a one percent determination of the primordial deuterium abundance. Combining our result with a big bang nucleosynthesis (BBN) calculation that uses the latest nuclear physics input, we find that the baryon density derived from BBN agrees to within $2 \sigma$ of the latest results from the Planck cosmic microwave background data.
\end{abstract}

Key words: cosmology: observations - cosmology: theory - primordial nucleosynthesis - quasars: absorption lines quasars: individual $(\mathrm{Q} 1243+307)$

\section{Introduction}

Modern cosmology is described by just six model parameters, all of which are known to within a few percent. This model provides a reliable description of the universe from seconds after the big bang until the present epoch. However, we know that the Standard Model of cosmology and particle physics is incomplete. For example, we have no definitive description of dark matter and dark energy, nor do we fully understand the properties of neutrinos. New physics beyond the Standard Model may be exposed by measuring the cosmological model parameters at high precision, and there are many teams that are searching for this new physics by studying the cosmic microwave background (CMB), weak and strong lensing, and by observing standard candles, rulers, and sirens, to name a few examples.

In recent years, there have also been several efforts to measure the chemical abundances of the elements that were made during the first minutes after the big bang, a process that is commonly referred to as "big bang nucleosynthesis" (BBN; for a general review of the subject, see Steigman 2007; Cyburt et al. 2016; Mathews et al. 2017). The abundances of the primordial elements - which include the isotopes of hydrogen, helium, and lithiumare sensitive to the physics of the early universe, and are therefore a tool that allows us to test the Standard Model. Moreover, measuring the abundances of these primordial elements currently provides our earliest test of the Standard Model.

\footnotetext{
* Based on observations collected at the W.M. Keck Observatory which is operated as a scientific partnership among the California Institute of Technology, the University of California, and the National Aeronautics and Space Administration. The Observatory was made possible by the generous financial support of the W.M. Keck Foundation.

${ }^{5}$ Royal Society University Research Fellow.
}

In order to reliably measure the primordial element abundances, we must first identify environments that are as close as possible to being pristine, and therefore still retain a primordial composition of the light elements. The best available measurements of the primordial element abundances come from different environments; conventionally, the mass fraction of ${ }^{4} \mathrm{He}\left(\mathrm{Y}_{\mathrm{P}}\right)$ is derived from the emission lines of nearby $\mathrm{H}$ II regions in metal-poor star-forming galaxies (Izotov et al. 2014; Aver et al. 2015), ${ }^{6}$ while the primordial ${ }^{7} \mathrm{Li}$ abundance is determined from the atmospheres of very metal-poor stars (Asplund et al. 2006; Aoki et al. 2009; Meléndez et al. 2010; Sbordone et al. 2010; Spite et al. 2015). At present, there are no reliable measurements of the primordial ${ }^{3} \mathrm{He}$ abundance; however, with future facilities this measurement may become possible (several different techniques are described by Bania et al. 2002; McQuinn \& Switzer 2009; Cooke 2015).

The only other primordial element that is accessible with current facilities is deuterium, which can be measured using gas clouds that are seen in absorption against the light of an unrelated background light source (typically, a quasar) (Adams 1976). Although this technique was proposed more than four decades ago, the first measurements were only achieved some 20 years later; even now, only a handful detections of the neutral deuterium (D I) absorption lines have been made (Burles \& Tytler 1998a, 1998b; Pettini \& Bowen 2001; O'Meara et al. 2001, 2006; Kirkman et al. 2003; Crighton et al. 2004; Pettini et al. 2008; Fumagalli et al. 2011; Noterdaeme et al. 2012; Pettini \& Cooke 2012; Cooke et al. 2014, 2016; Riemer-Sørensen et al. 2015, 2017; Balashev et al. 2016; Zavarygin et al. 2017). However, as discussed recently by Cooke et al. (2014), absorption line systems that

\footnotetext{
$6 \mathrm{Y}_{\mathrm{P}}$ can also be measured from the small-scale CMB temperature fluctuations (Planck Collaboration et al. 2016), albeit with lower precision.
} 
have $\mathrm{H}$ I column densities near the threshold of a damped Ly $\alpha$ system $\left(\mathrm{DLA} ; N(\mathrm{HI}) \simeq 10^{20.3} \mathrm{~cm}^{-2}\right)^{7}$ are the most suitable environments to precisely measure the primordial deuterium abundance, $(\mathrm{D} / \mathrm{H})_{\mathrm{P}}$ (see also Riemer-Sørensen et al. 2017). In this $\mathrm{H}$ I column density regime, the $\mathrm{H} \mathrm{I}$ Ly $\alpha$ transition exhibits Lorentzian damped wings that uniquely determine the total H I column density, while up to $\sim 10$ high order unsaturated D I lines are available to determine the total D I column density. Even among DLAs, only those that are kinematically quiescent are able to deliver a precise determination of $(\mathrm{D} / \mathrm{H})_{\mathrm{P}}$, since the D I lines need to be optically thin and unblended with nearby absorption lines. Empirically, it has been noted by several authors that DLAs with simple kinematics tend to be more common at the lowest metallicity (Ledoux et al. 2006; Murphy et al. 2007; Prochaska et al. 2008; Jorgenson et al. 2013; Neeleman et al. 2013; Cooke et al. 2015). Currently, there are just six systems which satisfy the above conditions, all of which have been homogeneously analyzed, as reported in previous papers of this series (Cooke et al. 2014, 2016).

The primordial deuterium abundance is inferred under the assumption that the ratio of deuterium to hydrogen atoms, $\mathrm{D} / \mathrm{H} \equiv N(\mathrm{DI}) / N(\mathrm{HI})$. There are several physical processes that potentially weaken the validity of this assumption, including: (1) The astration of deuterium as gas is cycled through generations of stars (Dvorkin et al. 2016; van de Voort et al. 2017, and the comprehensive list of references provided by Cyburt et al. 2016); (2) the relative ionization of deuterium and hydrogen in neutral gas (Savin 2002; Cooke \& Pettini 2016); and (3) the preferential depletion of deuterium onto dust grains (Jura 1982; Draine 2004, 2006). The first two physical processes are expected to alter the measured $\mathrm{D} / \mathrm{H}$ ratio by $\lesssim 0.1 \%$ when the metallicity is $\lesssim 1 / 100$ solar and the neutral hydrogen column density exceeds $10^{19} \mathrm{~cm}^{-2}$; this correction is an order of magnitude below the current measurement precision. The preferential depletion of deuterium onto dust grains, however, has not been modeled in detail in metalpoor DLAs.

Several studies have reported on the depletion of deuterium in the local interstellar medium (ISM) of the Milky Way (Wood et al. 2004; Prochaska et al. 2005; Linsky et al. 2006; Ellison et al. 2007; Lallement et al. 2008; Prodanović et al. 2010). However, the ISM of the Milky Way is relatively dust-rich compared with the metal-poor DLAs that are typically used to infer the primordial deuterium abundance. Observationally, metal-poor DLAs are not expected to contain a significant amount of dust (Murphy \& Bernet 2016); even the most refractory elements in the lowest-metallicity DLAs are hardly incorporated into dust grains (Pettini et al. 1997; Vladilo 2004; Akerman et al. 2005). However, Cooke et al. (2016) noted a subtle (but statistically insignificant) decline of the deuterium abundance with increasing metallicity, a trend that would be expected if deuterium were preferentially incorporated into dust grains.

Herein, we report a seventh high precision measurement of the deuterium abundance in one of the most pristine environments currently known, to assess whether or not the deuterium abundance depends on metallicity. The paper is organized as follows: in Section 2, we describe the observational procedure and the details of the data reduction process. The analysis technique

\footnotetext{
In this paper, we use the term "DLA" to represent any absorption line system with $N(\mathrm{H} \mathrm{I})>10^{20.3} \mathrm{~cm}^{-2}$ and "sub-DLA" for systems with $10^{19.0}<$ $N(\mathrm{H} \mathrm{I}) / \mathrm{cm}^{-2}<10^{20.3}$.
}

and the properties of the absorption system are then described in Section 3. In Section 4, we report our new $\mathrm{D} / \mathrm{H}$ abundance measurement of this system, and investigate the properties of our full sample. In Section 5, we deduce the primordial deuterium abundance, based on seven $\mathrm{D} / \mathrm{H}$ values, and provide new measurements of the cosmological baryon density, and effective number of neutrino species. Our conclusions are summarized in Section 6. All reported uncertainties represent $68 \%$ confidence intervals, unless otherwise stated.

\section{Observations and Data Reduction}

\subsection{Observational Data}

This paper presents an estimate of the primordial $\mathrm{D} / \mathrm{H}$ abundance using new, high quality data of a previously known sub-DLA at an absorber redshift $z_{\text {abs }} \simeq 2.5257$ toward the quasar $\mathrm{Q} 1243+307\left(z_{\mathrm{em}} \simeq 2.558\right.$, R.A. $=12^{\mathrm{h}} 46^{\mathrm{m}} 10^{\mathrm{s}} .9$, decl. $=+30^{\circ}$ $31^{\prime} 31$ "' 2; J2000). A measure of the deuterium abundance of this system was first reported by Kirkman et al. (2003), using data taken with the High Resolution Echelle Spectrometer (HIRES; Vogt et al. 1994) on the Keck I telescope during the years 1999-2000 (program IDs: U32H, U02H). These data consist of a total exposure time of $55,800 \mathrm{~s}$, divided into seven exposures, acquired with the previous generation HIRES detector; this detector had relatively low UV quantum efficiency and significantly higher read noise at the bluest wavelengths where the redshifted D I absorption lines are observed. For these reasons, ${ }^{8}$ we have re-observed Q1243+307 using the modern HIRES detector, which is considerably more sensitive at blue wavelengths.

Our observations (program ID: $\mathrm{N} 162 \mathrm{Hb}$ ) consisted of $3 \times$ $3600 \mathrm{~s}$ and $1 \times 3000 \mathrm{~s}$ exposures, and were carried out on 2016 March 30, in excellent seeing conditions ( 0 !! 6 full width at half maximum; FWHM), well matched to the chosen slit size (C1 decker, 0 "! $861 \times 7$ !"0). This decker provides a nominal spectral resolution of $R \simeq 48,000\left(v_{\mathrm{FWHM}} \simeq 6.25 \mathrm{~km} \mathrm{~s}^{-1}\right)$ for a uniformly illuminated slit. Using an exposure of a thoriumargon (ThAr) lamp, we directly measured the instrument FWHM to be $v_{\text {FWHM }}=6.28 \pm 0.02 \mathrm{~km} \mathrm{~s}^{-1}$ based on 2192 emission lines; throughout our analysis, we adopt this FWHM value. ${ }^{9}$ All science and calibration frames were binned $2 \times 2$ during read-out. The final combined signal-to-noise ratio $(\mathrm{S} / \mathrm{N})$ per $2.5 \mathrm{~km} \mathrm{~s}^{-1}$ pixel of our data near the observed wavelength $\lambda_{\text {obs }}=3215 \AA$ (i.e., the Lyman limit of the subDLA) is $\mathrm{S} / \mathrm{N} \simeq 25$. The $\mathrm{S} / \mathrm{N}$ is much higher at longer wavelengths, and reaches a maximum value of $\mathrm{S} / \mathrm{N} \simeq 80$ per $2.5 \mathrm{~km} \mathrm{~s}^{-1}$ pixel near the sub-DLA's Ly $\alpha$ absorption line.

Finally, a single exposure of length $3600 \mathrm{~s}$ was acquired with Keck+HIRES on 2006 June 2 (program ID: U152Hb, Lehner et al. 2014; O'Meara et al. 2015), using a nearly identical setup to our own observations (hereafter referred to as the KODIAQ data). We retrieved all of the aforementioned HIRES data of

\footnotetext{
8 This system was not analyzed in our previous work (Cooke et al. 2014), since the data were not publicly available at the time.

9 Ideally, the instrument FWHM should be determined using narrow telluric absorption lines, since the quasar was not uniformly illuminating the slit during the observations. Unfortunately, there are no telluric absorption bands covered by our spectrum, and we have therefore adopted the FWHM value of a uniformly illuminated slit. We note that this assumption should not affect our determination of $\mathrm{D} \mathrm{I} / \mathrm{H} \mathrm{I}$, because the equivalent width of an absorption line is invariant under convolution with the instrumental FWHM. As discussed in Section 3 , the equivalent widths of the weak D I absorption lines and the damped profile of the strong Ly $\alpha$ absorption line uniquely determine the D I and $\mathrm{H}$ I column densities, respectively.
} 
Table 1

Journal of Keck HIRES Observational Data Used in This Analysis

\begin{tabular}{|c|c|c|c|c|c|c|}
\hline Date & $\begin{array}{l}\text { Principal } \\
\text { Investigator }\end{array}$ & $\begin{array}{l}\text { Program } \\
\text { ID }\end{array}$ & $\begin{array}{l}\text { HIRES } \\
\text { Decker }\end{array}$ & $\begin{array}{c}v_{\mathrm{FWHM}} \\
\left(\mathrm{km} \mathrm{s}^{-1}\right)\end{array}$ & $\begin{array}{l}\text { Wavelength } \\
\text { Range }(\AA)\end{array}$ & $\begin{array}{c}\text { Exposure } \\
\text { Time (s) }\end{array}$ \\
\hline 1999 Apr 17, 18 & Tytler & $\mathrm{U} 32 \mathrm{H}$ & $\mathrm{C} 5$ & $7.99 \pm 0.02$ & $3190-4665$ & 23,400 \\
\hline 2000 Mar 13, 14 & Tytler & $\mathrm{U} 02 \mathrm{H}$ & C5 & $7.99 \pm 0.02$ & $3190-4665$ & 32,400 \\
\hline 2016 Mar 31 & Cooke & $\mathrm{N} 162 \mathrm{Hb}$ & $\mathrm{C} 1$ & $6.28 \pm 0.02$ & $3225-6085$ & 14,400 \\
\hline
\end{tabular}

Q1243+307 from the public Keck Observatory data archive. ${ }^{10}$ A summary of the data that are used in our analysis is provided in Table 1.

\subsection{Data Reduction Methods}

The modern HIRES data (program IDs: U152Hb, N162Hb) were reduced using the HIRES Redux package (Bernstein et al. 2015). ${ }^{11}$ We adopted the standard processing steps, including a bias level subtraction, correcting for the pixel-to-pixel variations and dividing by the blaze function of each echelle order. The echelle orders were traced using an exposure of a quartz lamp taken through the $\mathrm{C} 1$ decker (i.e., the same slit that was used to acquire the science frames). We employed an optimal sky subtraction and object extraction technique (Kelson 2003), and each pixel was assigned a wavelength using an exposure of a ThAr lamp that bracketed each science exposure.

At the time of our analysis, the HIRES Redux package was not able to reduce data acquired with the old HIRES detector (see, however, O'Meara et al. 2017). We therefore reduced the Kirkman et al. (2003) data (program IDs: U32H, U02H) using version 5.2.4 of the MAKEE data reduction pipeline, ${ }^{12}$ adopting a similar approach as that described by Suzuki et al. (2003). To summarize, we performed a bias subtraction, a flatfield and blaze correction, and traced the orders using an exposure of a quartz lamp taken through a pinhole decker. The data were wavelength calibrated using a ThAr lamp; we measured the widths of 1040 ThAr lines to be $v_{\mathrm{FWHM}}=7.99 \pm 0.02 \mathrm{~km} \mathrm{~s}^{-1}$, which is in good agreement with the nominal value of the instrument resolution $\left(v_{\mathrm{FWHM}}=8.0 \mathrm{~km} \mathrm{~s}^{-1}\right)$. For the analysis of the Kirkman et al. (2003) data set described below, we adopt our measured value of the FWHM.

All reduced data were corrected to the heliocentric frame of reference, and were converted to a vacuum wavelength scale. Using UVES_POPLER, ${ }^{13}$ we combined the exposures of each given data set to produce three separate spectra of Q1243+307: one spectrum of the Kirkman et al. (2003) data, one of the KODIAQ data, and the combined spectrum of our new data. As described in Section 3, all three of these spectra are kept separate from one another, but are analyzed simultaneously. For illustration purposes, in Figure 1 we show the complete combined spectrum of our new data (i.e., only the data acquired in 2016), flux calibrated with reference to the Kirkman et al. (2003) data.

\footnotetext{
${ }^{10}$ Available from: https://koa.ipac.caltech.edu/cgi-bin/KOA/nph-KOAlogin.

11 HIRES Redux is available from: http://www.ucolick.org/ xavier/HIRedux/.

12 MAKEE is available from: http://www.astro.caltech.edu/ tb/makee/.

13 UVES_POPLER is maintained by Michael T. Murphy, and is available from GitHub, via the following link: https://github.com/MTMurphy77/UVES_ popler.
}

\section{Analysis Method}

We now summarize the main aspects of our analysis method, which is identical to that described in our previous work (Cooke et al. 2014, 2016). We use the Absorption LIne Software (ALIS), which employs a $\chi$-squared minimization procedure to minimize the residuals between the data and a user-specified model, weighted by the inverse variance of the data. ${ }^{14}$

A key aspect of our analysis is that we simultaneously fit the emission spectrum of the quasar and the absorption due to the intervening absorption line system. This approach ensures that the final error on $\mathrm{D} / \mathrm{H}$ includes the uncertainty associated with the quasar continuum placement. We include all available information of the absorption system in our analysis, including the HI and DI Lyman series absorption lines and the unblended metal absorption lines. The continuum near each absorption line is fit during the minimization process assuming that it is described by a low order Legendre polynomial. Typically, the degree of the Legendre polynomial is $\lesssim 4$, except near the Ly $\alpha$ absorption line, where a Legendre polynomial of degree 8 is used. We also include a global model parameter that defines the zero-level of each data set, to account for small residuals in the background subtraction and/or partial covering of the background quasar by the foreground sub-DLA. All three data sets are analyzed at the same time to obtain a global best-fit model; we simultaneously fit the same absorption model to all three data sets, while allowing the model of the quasar continuum around every absorption line in each data set to be different. ${ }^{15} \mathrm{We}$ also include two fitting parameters to determine the global relative velocity shift between the three data sets, to account for instrumental artifacts in the wavelength calibration (e.g., Whitmore \& Murphy 2015).

As discussed in Section 1, accurate estimates of the primordial deuterium abundance are afforded by systems where the wings of the Ly $\alpha$ absorption line are damped by the Lorentzian term of the Voigt profile. In this regime, the damped wings uniquely determine the $\mathrm{H}$ I column density. For this reason, the data are most sensitive to $N(\mathrm{H} \mathrm{I})$ when the optical depth of the absorption profile is $\tau \gtrsim 0.7$ (i.e., where the residual intensity is $\lesssim 50 \%$ of the quasar continuum); for the sub-DLA toward Q1243+307, this corresponds to all pixels within $\pm 470 \mathrm{~km} \mathrm{~s}^{-1}$ of the H I Ly $\alpha$ line relative to the redshift defined by the narrow metal absorption lines. In our analysis, we opted to include all pixels that are within a velocity interval of $-470 \leqslant v / \mathrm{km} \mathrm{s}^{-1} \leqslant+580$ (see also Section 3.1). Any blends that are identified within this velocity

\footnotetext{
14 ALIS is available for download from GitHub: https://github.com/rcookeast/ALIS.

${ }^{15}$ There are two reasons why the emission profile near each absorption line may be different for the three data sets. First, the three data sets that are analyzed in this paper were taken at different epochs; the quasar continuum and emission lines may vary over the 16 year period spanned by the observations. Second, these data sets were acquired with different instrument configurations; the relative differences in the spectrograph efficiency as a function of wavelength can change the apparent level of the quasar continuum.
} 


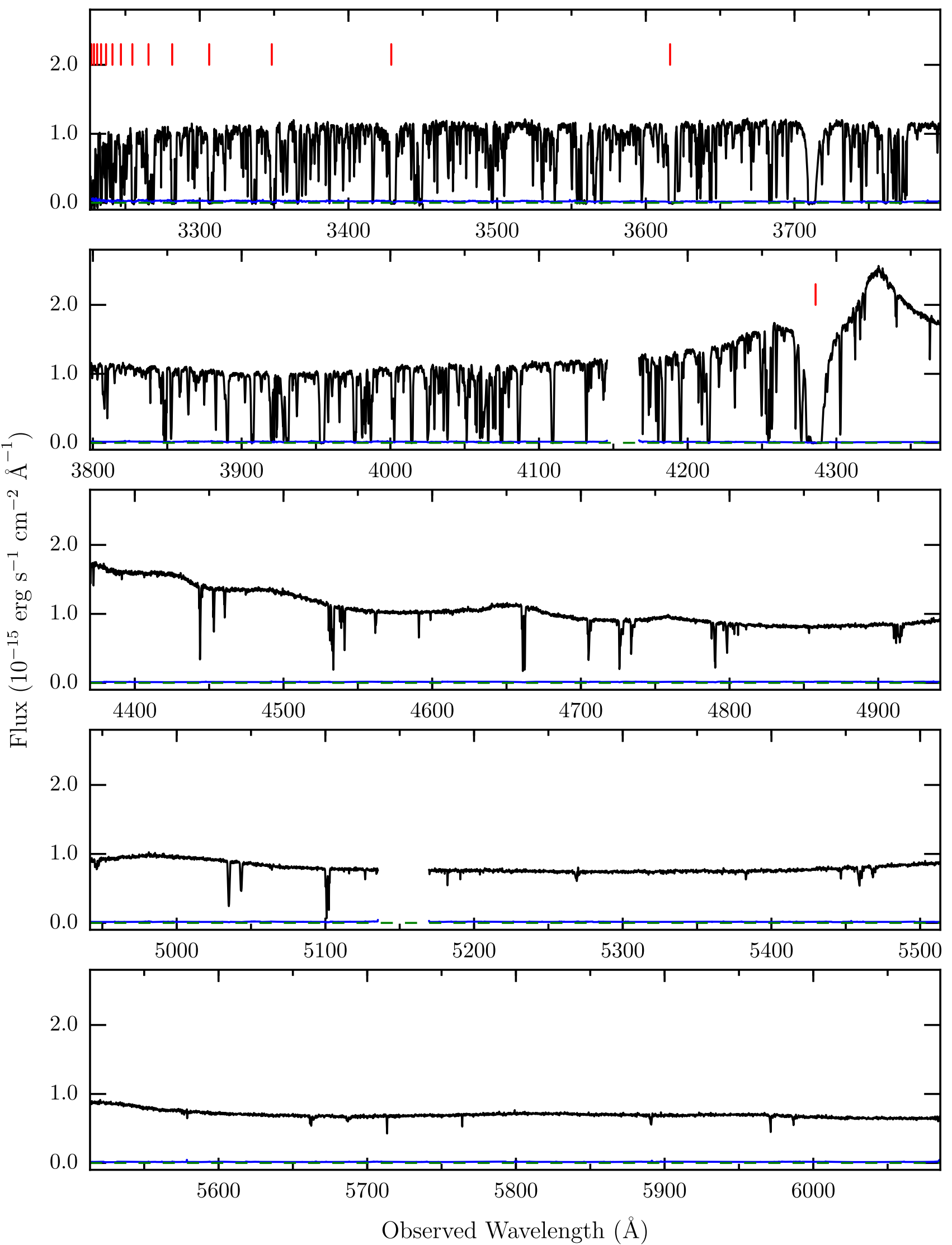

Figure 1. Final combined and flux-calibrated spectrum of Q1243+307 (black histogram) shown with the corresponding error spectrum (blue histogram) and zero level (green dashed line). The red tick marks above the spectrum indicate the locations of the Lyman series absorption lines of the sub-DLA at redshift $z_{\text {abs }}=2.52564$. Note the exquisite signal-to-noise ratio $(\mathrm{S} / \mathrm{N})$ of the combined spectrum, which varies from $\mathrm{S} / \mathrm{N} \simeq 80$ near the Ly $\alpha$ absorption line of the sub-DLA ( $\sim 4300 \AA$ ) to $\mathrm{S} / \mathrm{N} \simeq 25$ at the Lyman limit of the sub-DLA, near $3215 \AA$ in the observed frame. 

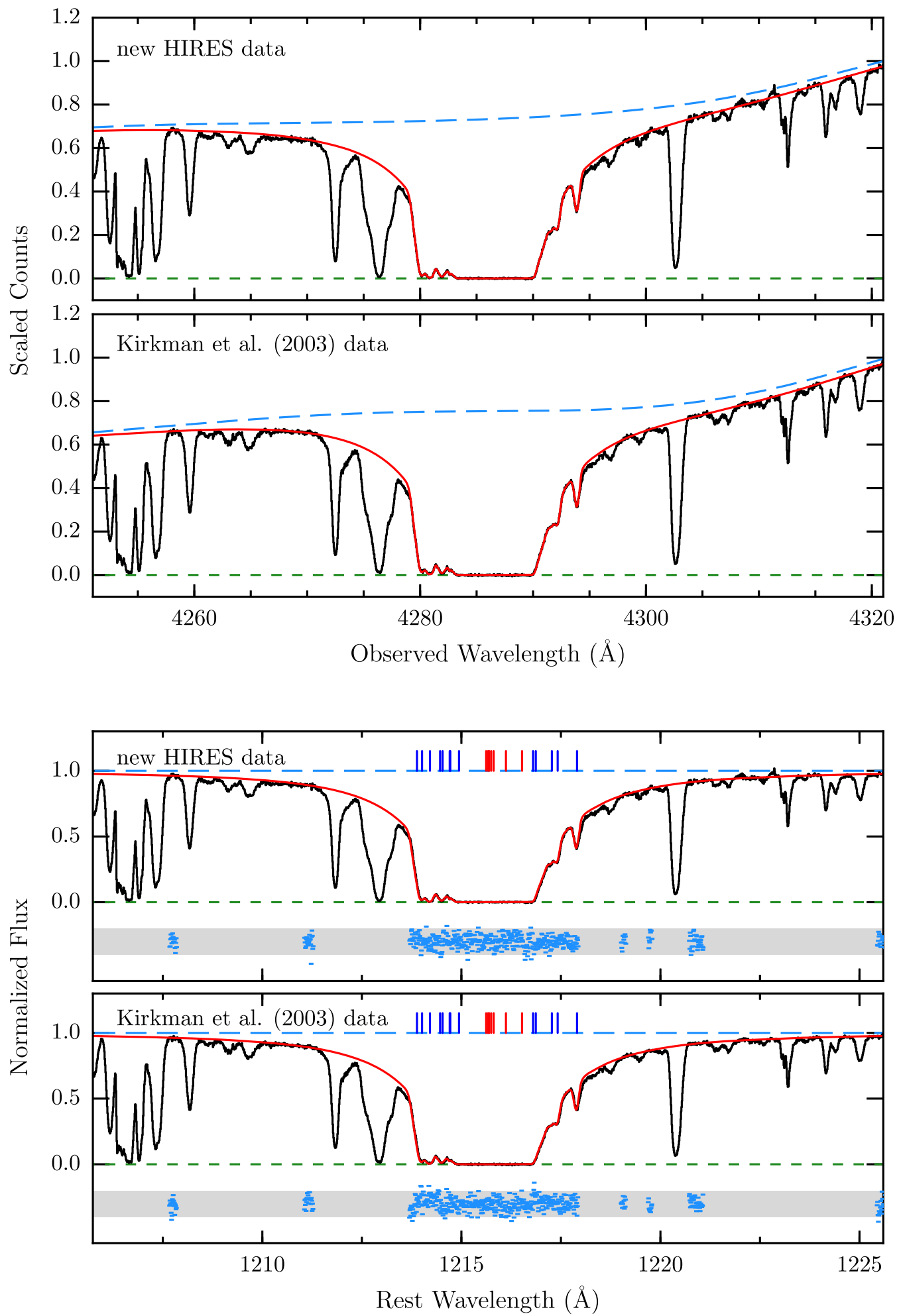

Figure 2. Ly $\alpha$ profile of the absorption system at $z_{\text {abs }}=2.52564$ toward the quasar Q1243+307 (black histogram) overlaid with the best-fitting model profile (red line), continuum (long dashed blue line), and zero-level (short dashed green line). The top panels show the raw, extracted counts scaled to the maximum value of the best-fitting continuum model. The bottom panels show the continuum normalized flux spectrum. The label provided in the top left corner of every panel indicates the source of the data. The blue points below each spectrum show the normalized fit residuals, (data-model)/error, of all pixels used in the analysis, and the gray band represents a confidence interval of $\pm 2 \sigma$. The $\mathrm{S} / \mathrm{N}$ is comparable between the two data sets at this wavelength range, but it is markedly different near the high order Lyman series lines (see Figures 4 and 5). The red tick marks above the spectra in the bottom panels show the absorption components associated with the main gas cloud (Components 2, 3, 4, 5, 6, 8, and 10 in Table 2), while the blue tick marks indicate the fitted blends. Note that some blends are also detected in Ly $\beta$-Ly $\epsilon$.

interval are modeled with a Voigt profile; outside this velocity interval we only include pixels in the $\chi$-squared minimization that are deemed by visual inspection to be free of unrelated absorption.
We present the data and best-fitting model profile of the Ly $\alpha$ absorption feature in Figure 2. The best-fitting model profile has an $\mathrm{HI}$ column density of $\log _{10} N(\mathrm{H} \mathrm{I}) / \mathrm{cm}^{-2}=19.761 \pm 0.026$, 


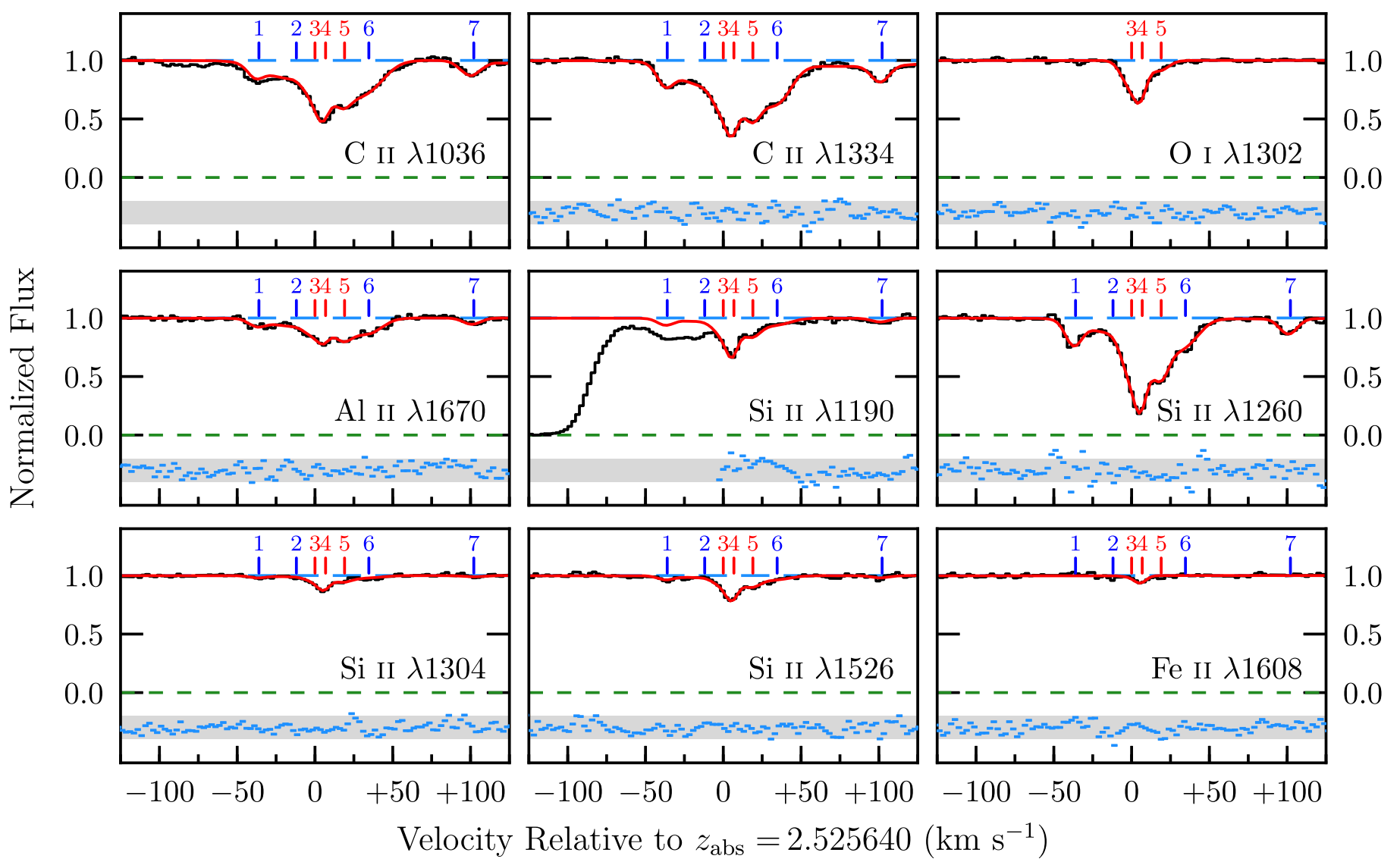

Figure 3. Metal absorption lines used in our analysis are shown as black histograms, overlaid with the best-fitting model (red line). The data and model are normalized to the best-fitting continuum model (long dashed blue line) and corrected for the fitted zero-level (short dashed green line). The red tick marks above each spectrum indicate the location of the absorption components seen in neutral gas (Components 3, 4, and 5 see Table 2), while the blue tick marks indicate the absorption components that are only seen in ionized gas (remaining components; see Table 2). The number above each tick mark indicates the Component Number, which is listed in the first column of Table 2. The blue points below each spectrum are the normalized fit residuals, (data-model)/error, of all pixels used in the analysis, and the gray band represents a confidence interval of $\pm 2 \sigma$. The different profiles exhibited by the neutral (O I) and singly ionized species (all remaining ions shown) are likely to be the result of ionized gas.

which is in good agreement with the corresponding estimate reported by Kirkman et al. (2003), $19.73 \pm 0.04$.

In Figure 3, we present the metal absorption lines in the $z_{\mathrm{abs}}=2.52564$ sub-DLA that were used in our analysis. We only show the new Keck HIRES data in this figure, but we note that our analysis includes all of the data that were described in Section 2.1. It is immediately obvious that the component structure of the metal absorption lines is complex, with the neutral species (e.g., O I) exhibiting a different structure to the singly ionized species (e.g., C II). This difference is probably due to the presence of ionized gas in the absorption system (see also Section 3.2). Table 2 lists full details of our absorption profile model which we now briefly summarize.

We assume that the gas in each absorption component is distributed according to a Maxwell-Boltzmann distribution, such that every component is represented by a Voigt profile characterized by a column density, a total Doppler parameter, and a redshift. We model the total Doppler parameter with a contribution from turbulent and thermal broadening:

$$
b_{\text {total }}^{2}=b_{\text {turb }}^{2}+b_{\text {therm }}^{2} \equiv b_{\text {turb }}^{2}+2 k_{\mathrm{B}} T_{\text {gas }} / m_{\text {ion }}
$$

where $T_{\text {gas }}$ is the gas temperature, $m_{\text {ion }}$ is the mass of the ion responsible for the absorption line, and $k_{\mathrm{B}}$ is the Boltzmann constant. As noted previously by Cooke et al. (2014), at the high quality of the data typically acquired for $\mathrm{D} / \mathrm{H}$ measurements, this model is too simplistic; in reality, there is a distribution of turbulence and gas temperature along the line-of-sight. To overcome this simplicity, we assume that all gas constituents share the same redshift and turbulent Doppler parameter, while the D I and H I thermal broadening components are fit separately. This prescription offers enough flexibility so that the total Doppler parameter of the H I, D I, and metal absorption lines can be determined almost independently. However, we emphasize that the weak D I absorption lines and the strong $\mathrm{H}$ I damped Ly $\alpha$ line do not depend on our choice to model the absorption lines as a Voigt profile. The D I column density only depends on the equivalent widths of several weak absorption lines, while the Lorentzian damped $\mathrm{HI} \operatorname{Ly} \alpha$ line uniquely determines the $\mathrm{HI}$ column density. The resulting D I/H I ratio is therefore unaffected by this assumption.

We find that the neutral O I absorption can be accurately described by three model components (denoted Components 3, 4 , and 5 in Table 2), which are indicated by the red tick marks above the spectrum in Figure 3. An additional five components are required to accurately represent the component structure of the ionized gas (Components 1, 2, 6, 7, and 9), shown as blue tick marks above the spectra in Figure $3 .{ }^{16}$ Finally, there are an

\footnotetext{
${ }^{16}$ In order to emphasize the structure of the absorption profile around the neutral absorption components, we have not shown the absorption of Component 9 (located at a velocity of $\Delta v=+198.1 \mathrm{~km} \mathrm{~s}^{-1}$ relative to Component 3) in Figure 3, since it is very weak.
} 
Table 2

Best-fitting Model Parameters of the Absorption System at $z_{\mathrm{abs}}=2.52564$ toward the QSO Q1243+307

\begin{tabular}{|c|c|c|c|c|c|c|c|c|c|c|c|}
\hline $\begin{array}{l}\text { Component } \\
\text { Number }\end{array}$ & $z_{\mathrm{abs}}$ & $\begin{array}{c}\Delta v \\
\left(\mathrm{~km} \mathrm{~s}^{-1}\right)\end{array}$ & $\begin{array}{c}b_{\text {turb }} \\
\left(\mathrm{km} \mathrm{s}^{-1}\right)\end{array}$ & $\begin{array}{c}\log _{10} N(\mathrm{H} \mathrm{I}) \\
\left(\mathrm{cm}^{-2}\right)\end{array}$ & $\log _{10}(\mathrm{D} \mathrm{I} / \mathrm{H} \mathrm{I})$ & $\begin{array}{c}\log _{10} N(\mathrm{C} \mathrm{II}) \\
\left(\mathrm{cm}^{-2}\right)\end{array}$ & $\begin{array}{c}\log _{10} N(\mathrm{O} \mathrm{I}) \\
\left(\mathrm{cm}^{-2}\right)\end{array}$ & $\begin{array}{c}\log _{10} N(\mathrm{Al} \text { II }) \\
\left(\mathrm{cm}^{-2}\right)\end{array}$ & $\begin{array}{c}\log _{10} N(\text { Si II }) \\
\left(\mathrm{cm}^{-2}\right)\end{array}$ & $\begin{array}{c}\log _{10} N(\mathrm{~S} \mathrm{II}) \\
\left(\mathrm{cm}^{-2}\right)\end{array}$ & $\begin{array}{c}\log _{10} N(\mathrm{Fe} \mathrm{II}) \\
\left(\mathrm{cm}^{-2}\right)\end{array}$ \\
\hline \multirow[t]{2}{*}{1} & 2.525216 & -36.1 & 5.9 & $\ldots^{\mathrm{b}}$ & $-4.622^{c}$ & 12.70 & $\ldots^{\mathrm{b}}$ & 10.96 & 11.87 & $\ldots^{\mathrm{b}}$ &.${ }^{\mathrm{b}}$ \\
\hline & \pm 0.000002 & \pm 0.9 & \pm 0.4 & & \pm 0.015 & \pm 0.04 & & \pm 0.09 & \pm 0.02 & & \\
\hline \multirow[t]{2}{*}{2} & 2.52550 & -11.9 & 23.0 & 17.23 & $-4.622^{\mathrm{c}}$ & 13.32 & $\ldots^{\mathrm{b}}$ & 11.60 & 12.14 & $\ldots^{\mathrm{b}}$ & $\ldots^{\mathrm{b}}$ \\
\hline & \pm 0.00003 & \pm 2.7 & \pm 0.8 & \pm 0.08 & \pm 0.015 & \pm 0.04 & & \pm 0.05 & \pm 0.04 & & \\
\hline \multirow[t]{2}{*}{3} & 2.52564 & 0.0 & 5.8 & 19.58 & $-4.622^{c}$ & 13.01 & 13.27 & 11.22 & 12.23 & $\ldots^{\mathrm{b}}$ & 11.73 \\
\hline & \pm 0.00001 & $\ldots$ & \pm 0.9 & \pm 0.07 & \pm 0.015 & \pm 0.11 & \pm 0.08 & \pm 0.12 & \pm 0.10 & & \pm 0.39 \\
\hline \multirow[t]{2}{*}{4} & 2.525720 & +6.8 & 3.4 & 19.08 & $-4.622^{c}$ & 13.21 & 13.31 & 11.28 & 12.55 & 12.59 & 12.39 \\
\hline & \pm 0.000002 & \pm 0.9 & \pm 0.3 & \pm 0.23 & \pm 0.015 & \pm 0.06 & \pm 0.08 & \pm 0.09 & \pm 0.05 & \pm 0.12 & \pm 0.07 \\
\hline \multirow[t]{2}{*}{5} & 2.525864 & +19.0 & 7.3 & 18.68 & $-4.622^{c}$ & 13.28 & 12.91 & 11.53 & 12.41 & $\ldots^{\mathrm{b}}$ & $\ldots^{\mathrm{b}}$ \\
\hline & \pm 0.000003 & \pm 0.9 & \pm 0.4 & \pm 0.17 & \pm 0.015 & \pm 0.04 & \pm 0.03 & \pm 0.05 & \pm 0.03 & & \\
\hline \multirow[t]{2}{*}{6} & 2.526047 & +34.6 & 12.3 & 16.86 & $-4.622^{c}$ & 13.34 & $\ldots{ }^{b}$ & 11.64 & 12.19 & $\ldots^{\mathrm{b}}$ & $\ldots^{\mathrm{b}}$ \\
\hline & \pm 0.000007 & \pm 1.0 & \pm 0.6 & \pm 0.04 & \pm 0.015 & \pm 0.03 & & \pm 0.04 & \pm 0.03 & & \\
\hline \multirow[t]{2}{*}{7} & 2.526841 & +102.1 & 6.8 & $\ldots^{\mathrm{b}}$ & $-4.622^{\mathrm{c}}$ & 12.69 & $\ldots^{\mathrm{b}}$ & 11.01 & 11.71 & $\ldots^{\mathrm{b}}$ & $\ldots^{\mathrm{b}}$ \\
\hline & \pm 0.000003 & \pm 0.9 & \pm 0.4 & & \pm 0.015 & \pm 0.04 & & \pm 0.05 & \pm 0.02 & & \\
\hline \multirow[t]{2}{*}{8} & 2.526943 & +110.8 & 25.9 & 16.242 & $-4.622^{\mathrm{c}}$ & $\ldots^{\mathrm{b}}$ & $\ldots^{\mathrm{b}}$ & $\ldots^{\mathrm{b}}$ & $\ldots \mathrm{b}$ & $\ldots^{\mathrm{b}}$ & $\ldots^{\mathrm{b}}$ \\
\hline & \pm 0.000003 & \pm 0.9 & \pm 0.2 & \pm 0.004 & \pm 0.015 & & & & & & \\
\hline \multirow[t]{2}{*}{9} & 2.52797 & +198.1 & 8.3 & $\ldots^{\mathrm{b}}$ & $-4.622^{c}$ & 11.57 & $\ldots^{\mathrm{b}}$ & 10.78 & 10.82 & $\ldots^{\mathrm{b}}$ & $\ldots^{\mathrm{b}}$ \\
\hline & \pm 0.00002 & \pm 1.9 & \pm 2.6 & & \pm 0.015 & \pm 0.36 & & \pm 0.13 & \pm 0.14 & & \\
\hline \multirow[t]{2}{*}{10} & 2.528115 & +210.5 & 27.6 & 16.365 & $-4.622^{c}$ & $\ldots^{\mathrm{b}}$ & $\ldots^{\mathrm{b}}$ & $\ldots^{\mathrm{b}}$ & $\ldots^{\mathrm{b}}$ & $\ldots^{\mathrm{b}}$ & $\ldots^{\mathrm{b}}$ \\
\hline & \pm 0.000002 & \pm 0.9 & \pm 0.1 & \pm 0.004 & \pm 0.015 & & & & & & \\
\hline \multirow[t]{2}{*}{$\operatorname{Total}^{\mathrm{d}}$} & & & & 19.761 & $-4.622^{\mathrm{c}}$ & 13.663 & 13.681 & 11.850 & 12.900 & 12.59 & 12.507 \\
\hline & & & & \pm 0.026 & \pm 0.015 & \pm 0.024 & \pm 0.011 & \pm 0.033 & \pm 0.011 & \pm 0.12 & \pm 0.085 \\
\hline
\end{tabular}

Notes. By comparing the relative widths of the metal and D I absorption lines, we determine the kinetic temperature of Component 3 and Component 4 to be $T_{\text {kin }}=8820 \pm 820 \mathrm{~K}$ and $T_{\text {kin }}=4100 \pm 2300 \mathrm{~K}$, respectively. For Components 5 and 6, we allowed the total Doppler parameters of the H I Lyman series absorption lines to vary independently of the metal absorption lines, which is equivalent to adding a thermal contribution to the line profiles. The resulting total Doppler parameters of the $\mathrm{H} \mathrm{I}$ lines of Components 5 and 6 are $b_{\mathrm{tot}}=14.6 \pm 0.6 \mathrm{~km} \mathrm{~s}^{-1}$ and $b_{\mathrm{tot}}=24.0 \pm 0.7 \mathrm{~km} \mathrm{~s}^{-1}$. The remaining absorption components are not sensitive to the relative contributions of turbulent and thermal broadening.

${ }^{\text {a }}$ The resulting $\chi$-squared/dof of this model is $12,115 / 13,697 \simeq 0.885$ (see footnote 19 ).

${ }^{\mathrm{b}}$ Absorption is undetected for this ion in this component.

${ }^{\mathrm{c}}$ Forced to be the same for all components.

${ }^{\mathrm{d}}$ The total column densities quoted are those in Components 3, 4, and 5, which together account for $99.5 \%$ of the total column density of neutral gas in this system. We note that the individual $\mathrm{H}$ I component column densities are strongly degenerate with each other due to the multi-component structure of the sub-DLA. Although the total $\mathrm{H}$ I column density is largely unaffected, the reported uncertainty of $N(\mathrm{H} \mathrm{I})$ reported here is likely overestimated. A more accurate estimate of the uncertainty on the total $\mathrm{H}$ I column density would be afforded by fitting directly to the total $\mathrm{H}$ I column density during the $\chi$-squared minimization, but this feature is not yet implemented in ALIS when the D I/H I ratio is forced to be the same in every component.

additional two components that are seen in the $\mathrm{HI}$ gas that are not seen in the low ion metal absorption lines (Components 8 and 10); these are also the two weakest $\mathrm{H}$ I components that are detected. We also point out that three of the absorption components (Components 1, 7, and 9) are detected in the metal absorption lines and do not have a discernible H I column density; it is difficult to resolve the H I absorption in these components due to their proximity to the stronger $\mathrm{HI}$ absorption exhibited by Components 3, 4, and 5 .

Due to the presence of ionized gas, we only provide an estimate of the oxygen abundance of this absorption system; $N(\mathrm{O} \mathrm{I}) / N(\mathrm{H} \mathrm{I})$ is considered a reliable measure of the $[\mathrm{O} / \mathrm{H}]$ abundance, ${ }^{17}$ since $\mathrm{OI}$ accurately traces the $\mathrm{HI}$ gas due to charge transfer reactions (Field \& Steigman 1971). Furthermore, we only consider the total column density of Components 3,4 , and 5 (the only components where $\mathrm{O}$ I is detected). Using a solar oxygen abundance of $\log _{10}(\mathrm{O} / \mathrm{H})_{\odot}+12=8.69$ (Asplund et al. 2009), we estimate an oxygen abundance

\footnotetext{
17 Throughout this paper, we adopt the notation $[\mathrm{X} / \mathrm{Y}]$ to represent the relative number density of elements $\mathrm{X}$ and $\mathrm{Y}$ on a logarithmic and solar abundance scale. Explicitly, $[\mathrm{X} / \mathrm{Y}]=\log _{10}(N(\mathrm{X}) / N(\mathrm{Y}))-\log _{10}(n(\mathrm{X}) / n(\mathrm{Y}))_{\odot}$.
}

$[\mathrm{O} / \mathrm{H}]=-2.769 \pm 0.028$, which compares well to that reported by Kirkman et al. (2003), of $[\mathrm{O} / \mathrm{H}]=-2.79 \pm 0.05$.

As in our previous analyses, we force all $\mathrm{H} \mathrm{I}$ components to share the same $N(\mathrm{D} \mathrm{I}) / N(\mathrm{H} \mathrm{I})$ ratio. The initial starting parameter value of the logarithmic $N(\mathrm{D} \mathrm{I}) / N(\mathrm{H} \mathrm{I})$ ratio is drawn from a uniform distribution over the range $(-4.7,-4.5)$. Our analysis uses a total of eight D I Lyman series lines, including Ly $\beta$, Ly $\epsilon-$ Ly 10 , and Ly $12 .{ }^{18}$ Since our data near the Lyman limit are of considerably higher $\mathrm{S} / \mathrm{N}$ than the data obtained by Kirkman et al. (2003; see Figures 4 and 5), we have identified several unrelated absorption line systems that are blended by chance with three of the DI absorption lines (Ly $\epsilon$, Ly7, and Ly9; see the Appendix); only one of these blends was discernible and accounted for in the lower $\mathrm{S} / \mathrm{N}$ data presented by Kirkman et al. (2003). This highlights the importance of obtaining high $\mathrm{S} / \mathrm{N}$ data down to the Lyman limit which, in this case, corresponds to an observed wavelength of $\sim 3215 \AA$. We have fully accounted for these blends by fitting the

\footnotetext{
${ }^{18}$ We do not include the Ly12 transition from the Kirkman et al. (2003) data in our analysis, since the data are noisy and the blue wing shows a different structure to the new HIRES data.
} 


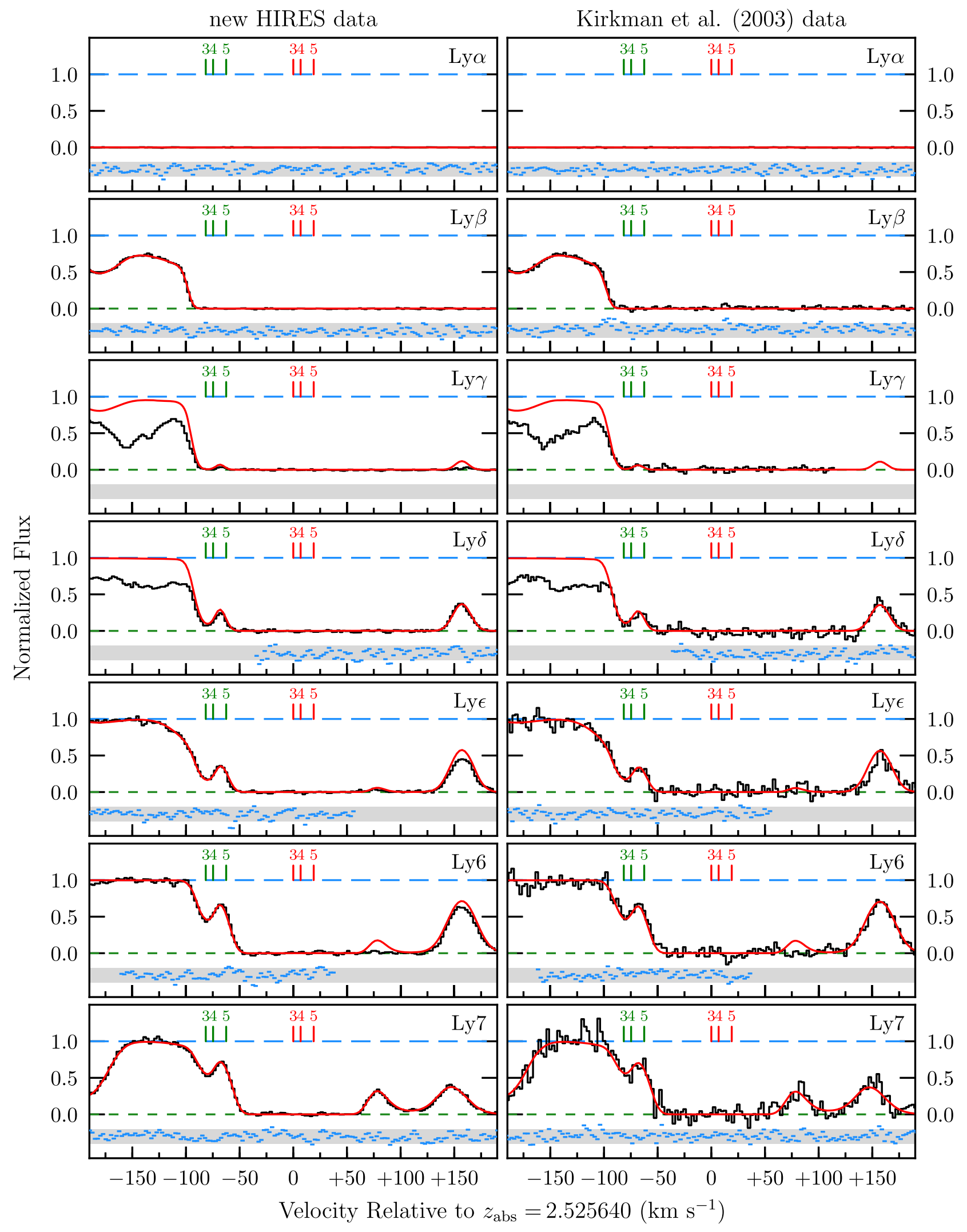

Figure 4. Lyman series lines of the absorption system at $z_{\text {abs }}=2.52564$ toward the QSO Q1243 +307 (black histograms) overlaid with the best-fitting model profile (red lines). The left panels display the newly acquired Keck HIRES data, which can be compared with the Kirkman et al. (2003) old Keck HIRES data shown in the right panels. In all panels, the data and models are normalized to the best-fitting continuum (long dashed blue lines), and the fitted zero level has been removed (short dashed green lines). The red and green tick marks above the spectrum indicate the locations of the three primary absorption components seen in $\mathrm{H}$ I and D I, respectively (denoted Components 3 , 4, and 5 in Table 2, as indicated above each tick mark). Although only three tick marks are shown, we note that the model profile presented in each panel (i.e., the red curve) includes all model components listed in Table 2. The blue points below each spectrum are the normalized fit residuals, (data-model)/error, of all pixels used in the analysis, and the gray band represents a confidence interval of $\pm 2 \sigma$. A label in the top right corner of every panel indicates the Lyman series transition shown. 


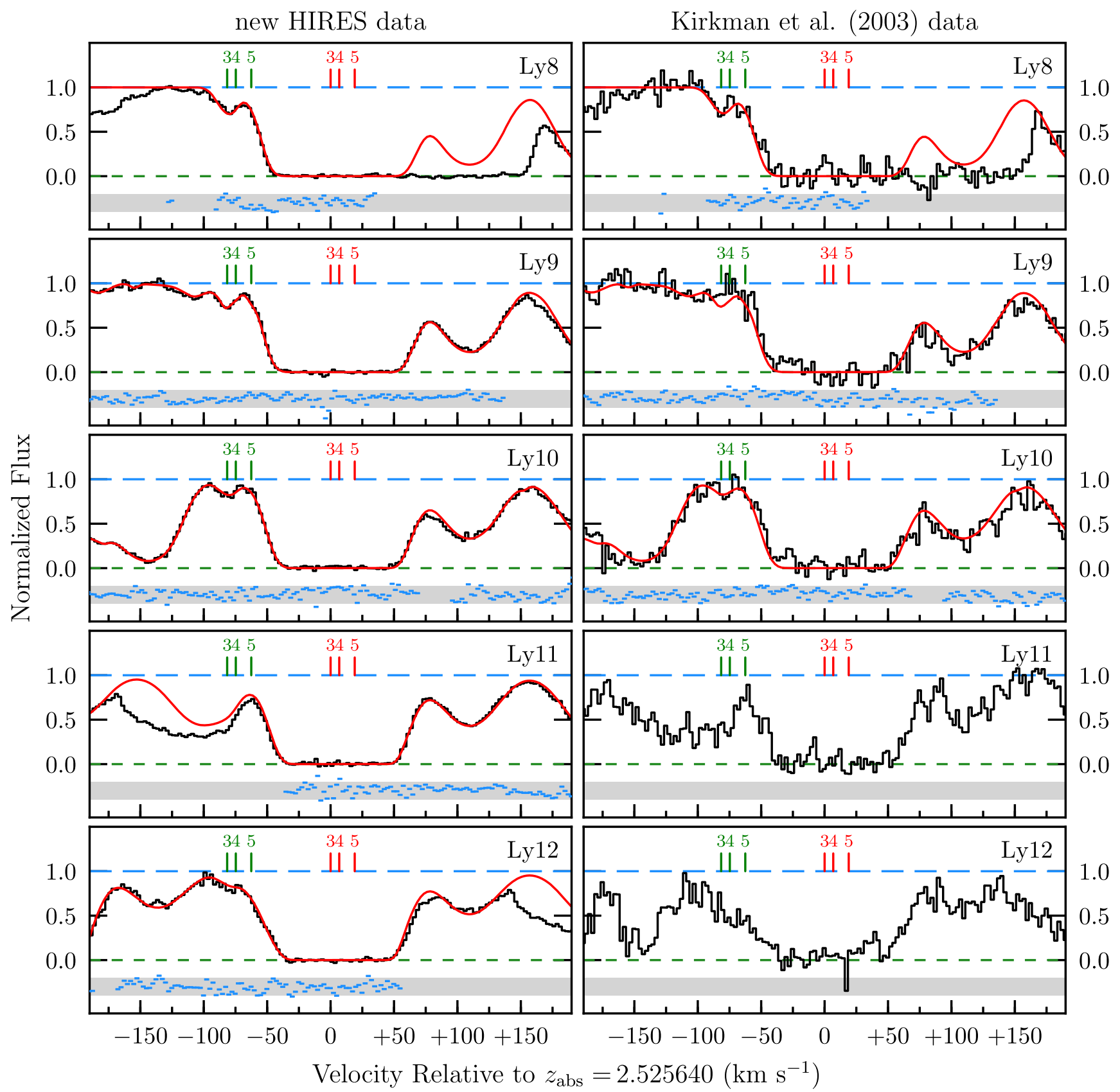

Figure 5. Same as Figure 4, for the higher order Lyman series lines. Both Ly11 and Ly12 from the Kirkman et al. (2003) data are not used in our analysis, but the data are shown here for reference without a model overplotted. We note that the Kirkman et al. (2003) data consist of a total exposure time of 55,800 s, while the new data we report here were obtained with a total exposure time of $13,800 \mathrm{~s}$. Even though the new data were taken with a quarter of the exposure time of the old data, they have considerably higher $\mathrm{S} / \mathrm{N}$, especially near the highest order Lyman series lines (corresponding to the weakest D I absorption lines) near an observed wavelength $\lambda_{\text {obs }}=3230 \AA$. This comparison offers a clear demonstration of the increased sensitivity made possible by the HIRES detector upgrade.

associated line profiles of each system, the details of which are provided in the Appendix. We present the best-fit model to the Lyman series lines in Figures 4 and 5.

Throughout the analysis described above, we adopt a blind analysis strategy, whereby the logarithmic $N(\mathrm{D} \mathrm{I}) / N(\mathrm{H}$ I $)$ ratio is not revealed until after the model fitting is complete. To ensure that the global minimum $\chi$-squared has been reached, we perform 2000 Monte Carlo simulations, where the starting parameters of each simulation correspond to the model fitting parameters perturbed by twice the covariance matrix. We also redraw a new logarithmic $N(\mathrm{D} \mathrm{I}) / N(\mathrm{H} \mathrm{I})$ ratio from a uniform distribution over the range $(-4.7,-4.5)$ for each simulation.
This process ensures that no memory of the starting parameters affects the final result. We then identify the realization that gives the minimum $\chi$-squared, unblind the logarithmic $N(\mathrm{D} \mathrm{I}) / N(\mathrm{H} \mathrm{I})$ ratio and refer to this as the "best-fitting" model throughout our analysis. We present the best-fitting model absorption profile parameters in Table 2. The resulting $\chi$-squared $/ \mathrm{dof}^{19}$ of this model is $12,115 / 13,697 \simeq 0.885$.

\footnotetext{
19 We note that our analysis does not account for correlations between neighboring spectral pixels, and therefore the reported $\chi$-squared value is likely underestimated, as discussed previously by Cooke et al. $(2014,2016)$.
} 


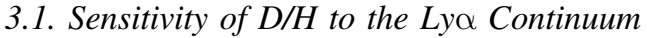

After the analysis was complete, we tested how the value and precision of $\mathrm{D} / \mathrm{H}$ were affected by the adopted fitting range around $\operatorname{Ly} \alpha$. We increased the fitted range to include all pixels and blends within $\pm 1250 \mathrm{~km} \mathrm{~s}^{-1}$ relative to the sub-DLA's redshift (i.e., all pixels with a residual intensity less than $90 \%$ of the quasar continuum). The central value of $\mathrm{D} / \mathrm{H}$ was unchanged and the error was increased by just $\sim 2.7 \%$ (from \pm 0.0150 to \pm 0.0154 in the $\log$; see Equation (2)), confirming that the final precision of $\mathrm{D} / \mathrm{H}$ is relatively insensitive to the pixels outside of the original velocity window (i.e., beyond $-470 \leqslant v / \mathrm{km} \mathrm{s}^{-1} \leqslant+580$ ).

\subsection{Sensitivity of $D / H$ to the Component Structure}

Subsequent to unblinding, we appreciated that some of the "satellite" absorption components of the C II $\lambda 1334$ and Si II $\lambda 1260$ absorption lines (in particular, Components 1 and 6) were underfit. We therefore included additional absorption components in the metal lines to test whether the value or error of the D I/H I value reported here were affected. By including an additional four absorption components (at velocities -38.8, $-5.8,+25.2$, and $45.7 \mathrm{~km} \mathrm{~s}^{-1}$ relative to Component 3 ), we found that the resulting $\chi$-squared was significantly improved $(11972 / 13678 \simeq 0.875)$. The central $\mathrm{D} / \mathrm{H}$ value of this model did not change, and the uncertainty increased by $6.4 \%$ (from \pm 0.015 to \pm 0.016 in the $\log$; see Equation (2)). This is a negligible increase, and is not reflected in the reported error budget on D I/H I.

\section{The Precision Sample}

\subsection{Sample Definition}

As outlined by Cooke et al. (2014), an absorption line system that meets the following selection criteria is almost ideal for obtaining a high precision measurement of the deuterium abundance. Specifically, all $\mathrm{D} / \mathrm{H}$ measures considered in this paper have: (1) an H I column density in excess of $10^{19} \mathrm{~cm}^{-2}$; in this column density regime, the $\operatorname{Ly} \alpha$ absorption line is damped by the Lorentzian term of the Voigt profile and a unique value of $N(\mathrm{H} \mathrm{I})$ can be determined from the wings of the line profile; (2) a $\operatorname{Ly} \alpha$ profile that is not severely blended by contaminating absorption; (3) at least two unblended and optically thin DI transitions from which the total column density of neutral deuterium can be determined; (4) data that were acquired with a high resolution echelle spectrograph, and of high S/N (>10 pixel $\left.^{-1}\right)$ near both Ly $\alpha$ and the weakest D I absorption line used in the analysis. We further add that all systems have been self-consistently analyzed by our group; including other recent determinations (Balashev et al. 2016; Riemer-Sørensen et al. 2017; Zavarygin et al. 2017) may introduce unaccounted for systematic errors because of the different analysis techniques adopted.

\subsection{A New Deuterium Abundance Measurement}

The absorption system at $z_{\text {abs }}=2.52564$ toward the quasar Q1243+307 satisfies all of the above criteria, and we now include this new measurement to the Precision Sample of D/H measures. Using the analysis procedure described in Section 3, we determine the logarithmic ratio of neutral deuterium to neutral hydrogen atoms of this absorption system to be:

$$
\log _{10} N(\mathrm{D} \text { I }) / N(\mathrm{H} \mathrm{I})=-4.622 \pm 0.015 .
$$

This number compares very well with the central value reported by Kirkman et al. (2003), $\log _{10} N(\mathrm{D} \mathrm{I}) / N(\mathrm{H} \mathrm{I})=-4.617_{-0.048}^{+0.058}$. The factor of 3.5 improvement on the $N(\mathrm{D} \mathrm{I}) / N(\mathrm{H} \mathrm{I})$ measurement precision that we report here is largely due to the much higher $\mathrm{S} / \mathrm{N}$ of the new data near the highest order Lyman series lines, compared to the Kirkman et al. (2003) data. It is reassuring that the central values reported by both analyses are mutually consistent.

The $N(\mathrm{DI}) / N(\mathrm{HI})$ value found here is consistent with the previous measures by Cooke et al. (2014, 2016), which are all collected in Table 3. This new $N(\mathrm{D} \mathrm{I}) / N(\mathrm{H} \mathrm{I})$ value comes from one of the lowest metallicity systems currently known, making it a key measurement to assess whether or not $N(\mathrm{D} \mathrm{I}) / N(\mathrm{H} \mathrm{I})$ varies with metallicity. We note that the estimated $N(\mathrm{D}$ I $) / N(\mathrm{H}$ I $)$ value of this system is the lowest of the seven systems analyzed by our group, and is derived from one of the lowest H I column density absorbers that we have considered so far. Despite the relatively low HI column density, we emphasize that the expected ionization correction is $<0.001$ dex when the $\mathrm{H}$ I column density is $10^{19.76}$ atoms cm ${ }^{-2}$ (Cooke \& Pettini 2016).

The Precision Sample of $\mathrm{D} / \mathrm{H}$ measurements is shown in the left and right panels of Figure 6 as a function of metallicity and H I column density, respectively. We first draw attention to the subtle decrease of $\mathrm{D} / \mathrm{H}$ with increasing metallicity suggested by the six blue symbols in Figure 6 (see also Cooke et al. 2016). The new measurement that we report here (indicated by the green symbol in Figure 6) does not support this trend. There is also no apparent trend of $\mathrm{D} / \mathrm{H}$ with $\mathrm{H}$ I column density. In what follows, we therefore assume that $N(\mathrm{D} \mathrm{I}) / N(\mathrm{H} \mathrm{I}) \equiv \mathrm{D} / \mathrm{H}$.

Table 3

Precision D/H Measures Considered in This Paper

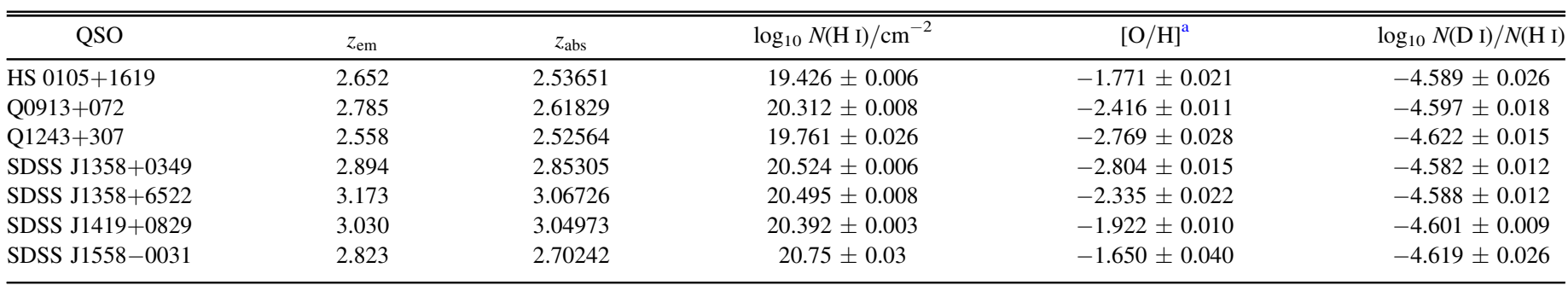

Note.

${ }^{\mathrm{a}}$ We adopt the solar value $\log _{10}(\mathrm{O} / \mathrm{H})+12=8.69$ (Asplund et al. 2009). 

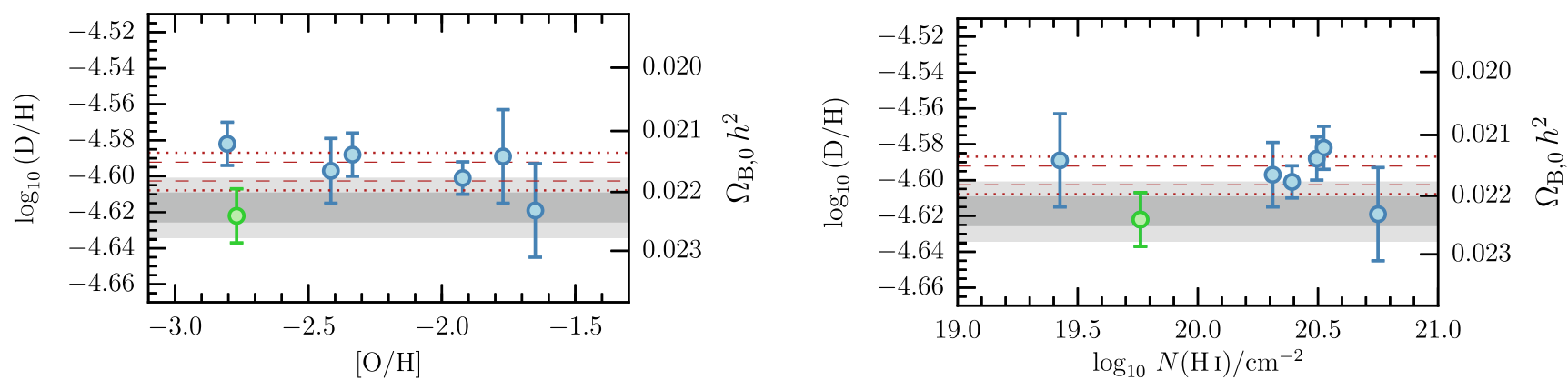

Figure 6. Our sample of seven high precision $\mathrm{D} / \mathrm{H}$ measures (symbols with error bars); the green symbol represents the new measure that we report here. The weighted mean value of these seven measures is shown by the red dashed and dotted lines, which represent the $68 \%$ and $95 \%$ confidence levels, respectively. The left and right panels show the dependence of $\mathrm{D} / \mathrm{H}$ on the oxygen abundance and neutral hydrogen column density, respectively. Assuming the Standard Model of cosmology and particle physics, the right vertical axis of each panel shows the conversion from $\mathrm{D} / \mathrm{H}$ to the universal baryon density. This conversion uses the Marcucci et al. (2016) theoretical determination of the $d(p, \gamma)^{3} \mathrm{He}$ cross-section. The dark and light shaded bands correspond to the $68 \%$ and $95 \%$ confidence bounds on the baryon density derived from the CMB (Planck Collaboration et al. 2016).

\subsection{Intrinsic Scatter}

Even though the seven measurements considered here show no apparent trend with metallicity or $\mathrm{H}$ I column density, there may still be an intrinsic scatter of these $\mathrm{D} / \mathrm{H}$ measurements that could be due to systematics that are currently unaccounted for. Such an "excess" dispersion in D/H abundance measurements was originally noted by Steigman (2001) for an earlier, and more heterogeneous, sample of $\mathrm{D} / \mathrm{H}$ values. Indeed, a simple $\chi^{2}$ test reveals that these seven measures are statistically consistent (i.e., within $2 \sigma$ ) of being drawn from a constant $\mathrm{D} / \mathrm{H}$ value. This suggests that the intrinsic scatter among the measurements must be low, and we now explore this in further detail.

Suppose that each measured $\mathrm{D} / \mathrm{H}$ value, $d_{i}$, with uncertainty $\sigma_{i}$ has a corresponding "true" value, $d_{\mathrm{T}}$. The probability that a given observation arises from the true value is given by

$$
\operatorname{Pr}\left(d_{i} \mid d_{\mathrm{T}}\right)=\frac{1}{\sqrt{2 \pi} \sigma_{i}} \exp \left(-\frac{\left(d_{i}-d_{\mathrm{T}}\right)^{2}}{2 \sigma_{i}^{2}}\right) .
$$

Similarly, if the true values are drawn from an "intrinsic" distribution with central value $\mathrm{DH}_{\mathrm{P}}$ and scatter $\sigma$, the probability that a true value is drawn from the intrinsic distribution is

$$
\operatorname{Pr}\left(d_{\mathrm{T}} \mid \mathrm{DH}_{\mathrm{P}}\right)=\frac{1}{\sqrt{2 \pi} \sigma} \exp \left(-\frac{\left(d_{\mathrm{T}}-\mathrm{DH}_{\mathrm{P}}\right)^{2}}{2 \sigma^{2}}\right) .
$$

Therefore, the probability of obtaining a measured $\mathrm{D} / \mathrm{H}$ value, $d_{i}$, given our intrinsic model is found by integrating over all possible true values

$$
\begin{aligned}
& \operatorname{Pr}\left(d_{i} \mid \mathrm{DH}_{\mathrm{P}}\right)=\int_{-\infty}^{+\infty} \operatorname{Pr}\left(d_{i} \mid d_{\mathrm{T}}\right) \operatorname{Pr}\left(d_{\mathrm{T}} \mid \mathrm{DH}_{\mathrm{P}}\right) \mathrm{d} d_{\mathrm{T}} \\
& \operatorname{Pr}\left(d_{i} \mid \mathrm{DH}_{\mathrm{P}}\right)=\frac{1}{\sqrt{2 \pi\left(\sigma_{i}^{2}+\sigma^{2}\right)}} \exp \left(-\frac{\left(d_{i}-\mathrm{DH}_{\mathrm{P}}\right)^{2}}{2\left(\sigma_{i}^{2}+\sigma^{2}\right)}\right)
\end{aligned}
$$

and the log-likelihood function is then given by

$$
\mathcal{L}=\log \left[\prod_{i} \operatorname{Pr}\left(d_{i} \mid \mathrm{DH}_{\mathrm{P}}\right)\right] .
$$

Using a brute force method, we solve for the parameter values $\left(\mathrm{DH}_{\mathrm{P}}\right.$ and $\left.\sigma\right)$ that maximize the likelihood function in Equation (6), based on the seven measures listed in Table 3.
The maximum likelihood parameter values are

$$
\begin{gathered}
\mathrm{DH}_{\mathrm{P}}=-4.5976 \pm 0.0072 \\
\sigma \leqslant 0.027 \quad(95 \% \text { confidence }) .
\end{gathered}
$$

Note that the intrinsic dispersion, $\sigma$, has a maximum likelihood value of zero; we therefore quote a $2 \sigma$ upper limit. The above likelihood analysis indicates that there is very little intrinsic scatter in our defined sample of consistently analyzed $\mathrm{D} / \mathrm{H}$ measures. We therefore speculate that the original excess scatter noted by Steigman (2001) is probably due to a combination of the different analysis techniques employed by different authors and the use of absorption line systems that were not well-suited for measuring $\mathrm{D} / \mathrm{H}$. Together, these factors probably resulted in underestimates of the true errors in the values of $\mathrm{D} / \mathrm{H}$ reported.

\section{Cosmological Consequences}

\subsection{The Primordial Deuterium Abundance}

Based on the analysis above, we conclude that the seven $\mathrm{D} / \mathrm{H}$ measurements considered here are drawn from the same value, and a weighted mean of these measures gives our best estimate of the primordial deuterium abundance: ${ }^{20}$

$$
\log _{10}(\mathrm{D} / \mathrm{H})_{\mathrm{P}}=-4.5974 \pm 0.0052
$$

or, expressed as a linear quantity:

$$
10^{5}(\mathrm{D} / \mathrm{H})_{\mathrm{P}}=2.527 \pm 0.030 \text {. }
$$

This value corresponds to a $\sim 1 \%$ determination of the primordial deuterium abundance, and is shown in Figure 6 by the dashed and dotted horizontal lines to represent the $68 \%$ and $95 \%$ confidence regions, respectively. Our determination of the primordial deuterium abundance quoted here has not changed much from our previous estimate in Cooke et al. (2016); as discussed above, the new value is in mutual agreement with the previous six measures and is of comparable precision. We therefore conclude that the primordial deuterium abundance quoted here is robust.

\footnotetext{
20 These values and their errors are unaffected by the small error increases resulting from the changes to our fitting procedure, as discussed in Sections 3.1 and 3.2 .
} 


\subsection{Testing the Standard Model}

In order to compare this measurement to the latest Planck CMB results, we must first convert our estimate of $(\mathrm{D} / \mathrm{H})_{\mathrm{P}}$ to the baryon-to-photon ratio, $\eta$. To do this, we use the BBN calculations described by Cooke et al. (2016; see also Nollett \& Burles 2000; Nollett \& Holder 2011), assuming the Marcucci et al. (2016) $d(p, \gamma)^{3} \mathrm{He}$ reaction rate. For the case of the Standard Model, we deduce a baryon-to-photon ratio of

$$
10^{10} \eta \equiv \eta_{10}=5.931 \pm 0.051
$$

which includes the uncertainty of the nuclear data that are used as input to the BBN calculations.

We can now convert this value of the baryon-to-photon ratio into an estimate of the cosmic density of baryons using the formula $\eta_{10}=(273.78 \pm 0.18) \times \Omega_{\mathrm{B}, 0} h^{2} \quad($ Steigman 2006) which, for the Standard Model, gives the value:

$$
100 \Omega_{\mathrm{B}, 0} h^{2}(\mathrm{BBN})=2.166 \pm 0.015 \pm 0.011
$$

where the first error term includes the uncertainty in the measurement of $(\mathrm{D} / \mathrm{H})_{\mathrm{P}}$, and the second error term provides the uncertainty in the BBN calculations.

The BBN inferred value of the cosmic baryon density is somewhat lower than the $\mathrm{CMB}$ value (Planck Collaboration et al. 2016; see the gray bands in Figure 6): ${ }^{21}$

$$
100 \Omega_{\mathrm{B}, 0} h^{2}(\mathrm{CMB})=2.226 \pm 0.023
$$

This difference corresponds to a $2 \sigma$ discrepancy. As discussed by Cooke et al. (2016), there is still some tension between the computed and the empirically measured $d(p, \gamma)^{3} \mathrm{He}$ crosssection that is used as input into the BBN calculations. Adopting the empirically measured $d(p, \gamma)^{3} \mathrm{He}$ cross-section proposed by Adelberger et al. (2011), we estimate a cosmic baryon abundance of

$$
100 \Omega_{\mathrm{B}, 0} h^{2}(\mathrm{BBN})=2.235 \pm 0.016 \pm 0.033 .
$$

where the error terms have the same meaning as in Equation (12). This value is in better agreement with the CMB measurement. In the near future, we expect to hear the results of a $d(p, \gamma)^{3} \mathrm{He}$ measurement campaign from the Laboratory for Underground Nuclear Astrophysics (LUNA; Gustavino 2014; Kochanek 2016), which will provide new insight to this important $\mathrm{BBN}$ reaction rate.

An alternative possibility to bring closer together the BBN and $\mathrm{CMB}$ determinations of $\Omega_{\mathrm{B}, 0} h^{2}$ is to consider simple extensions to the Standard Model, such as a change in the expansion rate, parameterized by the effective number of neutrino species, $N_{\text {eff }}$. In Figure 7, we present the confidence contours of the baryon density and the effective number of neutrino families for the $(\mathrm{D} / \mathrm{H})_{\mathrm{P}}$ estimate that we report here (blue band) ${ }^{22}$ in addition to the Planck CMB results (gray ellipse). The combined confidence contours are displayed in red, where the central value and uncertainty of these parameters are (95\% confidence limits):

$$
100 \Omega_{\mathrm{B}, 0} h^{2}=2.237 \pm 0.070
$$

\footnotetext{
${ }^{21}$ The quoted value of $\Omega_{\mathrm{B}, 0} h^{2}(\mathrm{CMB})$ corresponds to the Planck TT+lowP +lensing analysis, listed in the second data column of Table 4 from Planck Collaboration et al. (2016). As demonstrated by these authors, the CMB value of $\Omega_{\mathrm{B}, 0} h^{2}$ is robust to simple extensions of the base $\Lambda \mathrm{CDM}$ model.

${ }^{22}$ Assuming the Marcucci et al. (2016) $d(p, \gamma)^{3} \mathrm{He}$ reaction rate.
}

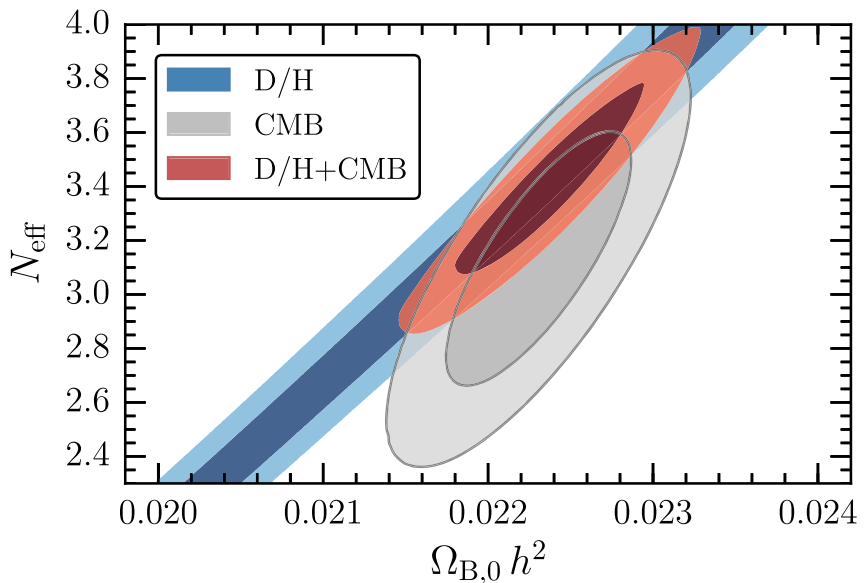

Figure 7. Comparing the expansion rate (parameterized by $N_{\text {eff }}$ ) and the cosmic density of baryons $\left(\Omega_{\mathrm{B}, 0} h^{2}\right)$ from BBN (blue contours) and CMB (gray contours). The red contours indicate the combined confidence bounds. The dark and light shades illustrate the $68 \%$ and $95 \%$ confidence contours, respectively.

$$
N_{\text {eff }}=3.41 \pm 0.45
$$

where the Standard Model value of the effective number of neutrino species is $N_{\text {eff }}=3.046$ (Mangano et al. 2005; see also Grohs et al. 2015). We therefore conclude that our results are consistent (within 2 $\sigma$ ) with the Standard Model of cosmology and particle physics.

\section{Summary and Conclusions}

We have presented a reanalysis of the $z_{\mathrm{abs}}=2.52564$ absorption system along the line-of-sight to Q1243+307 using data previously reported by Kirkman et al. (2003), combined with new data acquired with the Keck HIRES echelle spectrograph, to deduce the deuterium abundance of an extremely metal-poor gas cloud. Our conclusions are summarized as follows:

(i) Using the upgraded, ultraviolet sensitive detector on the HIRES echelle spectrograph, we have obtained exquisite, high signal-to-noise data down to the Lyman limit of Q1243+307 near the observed wavelength $\lambda_{\text {obs }}=3215 \AA$. Combined with archival data, we estimate the oxygen abundance of this absorber to be $[\mathrm{O} / \mathrm{H}]=-2.769 \pm$ 0.028 , which is among the lowest metallicity environment where the deuterium abundance has been measured.

(ii) On the basis of eight D I Lyman series absorption lines, we infer that the deuterium abundance of this system is $\log _{10} N(\mathrm{D} \mathrm{I}) / N(\mathrm{H} \mathrm{I})=-4.622 \pm 0.015$, which is in excellent agreement with the value reported by Kirkman et al. $\left(2003, \log _{10} N(\mathrm{D} \mathrm{I}) / N(\mathrm{H} \mathrm{I})=-4.617_{-0.048}^{+0.058}\right)$. Our measure has therefore improved the precision of this one measurement by a factor of $\sim 3.5$.

(iii) Combining our new measurement with the six homogeneously analyzed systems previously reported by our group, we use a maximum likelihood technique to determine the intrinsic dispersion of our $\mathrm{D} / \mathrm{H}$ sample. We find that the seven $\mathrm{D} / \mathrm{H}$ measures are consistent with being drawn from a constant value (i.e., no intrinsic dispersion). Thus, the excess dispersion of $\mathrm{D} / \mathrm{H}$ values previously recognized by Steigman (2001) can be attributed to the different analysis techniques adopted by different authors, and the use of absorption line systems that were not well-suited for precisely measuring 
the primordial abundance of deuterium. Together, these factors probably resulted in underestimates of the true errors in the values of $\mathrm{D} / \mathrm{H}$ reported.

(iv) We also find that these seven $\mathrm{D} / \mathrm{H}$ values do not correlate with $[\mathrm{O} / \mathrm{H}]$ or $N(\mathrm{H} \mathrm{I})$, strongly suggesting that our sample of $\mathrm{D} / \mathrm{H}$ measures corresponds to the primordial abundance of deuterium.

(v) Based on the seven systems analyzed by our group, we estimate that the primordial deuterium abundance is $\log _{10}(\mathrm{D} / \mathrm{H})_{\mathrm{P}}=-4.5974 \pm 0.0052$, or, expressed as a linear quantity $10^{5}(\mathrm{D} / \mathrm{H})_{\mathrm{P}}=2.527 \pm 0.030$. Thanks to modern instrumentation and a careful analysis, it is now possible to pin down the primordial abundance of deuterium with $\sim 1 \%$ precision, using just seven $\mathrm{D} / \mathrm{H}$ measures.

(vi) Using a suite of $\mathrm{BBN}$ calculations that use the latest nuclear physics input (previously described by Cooke et al. 2016), we estimate that the cosmic abundance of baryons is $100 \Omega_{\mathrm{B}, 0} h^{2}(\mathrm{BBN})=2.166 \pm 0.015 \pm 0.011$, where we separately quote the error associated with the measurement (former) and the BBN calculation (latter). This value is based on the $d(p, \gamma)^{3} \mathrm{He}$ reaction rate computed by Marcucci et al. (2016), and differs from the Planck CMB value by $2 \sigma$. Alternatively, using an empirically determined $d(p, \gamma)^{3} \mathrm{He}$ reaction rate, we estimate a baryon density of $100 \Omega_{\mathrm{B}, 0} h^{2}(\mathrm{BBN})=2.235 \pm 0.016 \pm 0.033$, which is in better agreement with the $\mathrm{CMB}$, albeit with larger errors.

(vii) We also perform a joint analysis of $\mathrm{D} / \mathrm{H}$ and the Planck CMB data to place a bound on the effective number of neutrino species. Our joint constraints on the cosmic baryon abundance and effective number of neutrino families are $100 \Omega_{\mathrm{B}, 0} h^{2}=2.237 \pm 0.070$ and $N_{\text {eff }}=$ $3.41 \pm 0.45$, respectively ( $95 \%$ confidence).

Given that the CMB is now limited by cosmic variance at scales $1 \lesssim 10^{3}$-the multipole regime where the temperature fluctuations are very sensitive to the baryon density-it will become increasingly difficult to significantly improve the precision of $\Omega_{\mathrm{B}, 0} h^{2}$ derived from the CMB. Based on just seven determinations of the deuterium abundance of near-pristine gas clouds, we have reached a one percent precision on the primordial deuterium abundance, corresponding to a sub-percent level precision on $\Omega_{\mathrm{B}, 0} h^{2}$; this level of precision is comparable to, or somewhat better than, that reached by the latest $\mathrm{CMB}$ constraints. In addition, there are exciting opportunities in the immediate future to further increase the statistics of $\mathrm{D} / \mathrm{H}$ with the The Echelle SPectrograph for Rocky Exoplanet and Stable Spectroscopic Observations (ESPRESSO) spectrograph on the European Southern Observatory Very Large Telescope, and potentially in the longer term with the 30-40 m class telescopes.

We are grateful to the staff astronomers at Keck Observatory for their assistance with the observations, and to Jason X. Prochaska, Tom Barlow, and Michael T. Murphy for providing some of the software that was used to reduce the data. We also thank an anonymous referee who provided helpful suggestions that improved the presentation of this work, following a referee who was unable to respond in a timely manner. During this work, R.J.C. was supported by a Royal Society University Research Fellowship. R.J.C. acknowledges support from STFC (ST/L00075X/1, ST/P000541/1). C.C.S. has been supported by grant AST-1313472 from the U.S. NSF. This research was also supported by a NASA Keck PI Data Award, administered by the NASA Exoplanet Science Institute.
Data presented herein were obtained at the W. M. Keck Observatory from telescope time partially allocated to the National Aeronautics and Space Administration through the agency's scientific partnership with the California Institute of Technology and the University of California. The Observatory was made possible by the generous financial support of the W. M. Keck Foundation. We thank the Hawaiian people for the opportunity to observe from MaunaKea; without their hospitality, this work would not have been possible. This work used the DiRAC Data Centric system at Durham University, operated by the Institute for Computational Cosmology on behalf of the STFC DiRAC HPC Facility (http://www.dirac.ac.uk). This equipment was funded by BIS National E-infrastructure capital grant ST/K00042X/1, STFC capital grant ST/H008519/1, and STFC DiRAC Operations grant ST/K003267/1 and Durham University. DiRAC is part of the National E-Infrastructure. This research has made use of NASA's Astrophysics Data System. R.J.C. thanks S.O.C. for her impeccable timing and invaluable insight.

Facility: Keck(HIRES).

Software: Astropy (Astropy Collaboration et al. 2013), Matplotlib (Hunter 2007), NumPy (van der Walt et al. 2011).

\section{Appendix \\ Accounting for Line Blending of the D I Lyman Series Profiles}

In this appendix, we present the model profiles of several unrelated absorption systems that are coincident (by chance) with the Lyman series absorption lines of the sub-DLA at $z_{\text {abs }}=2.52564$. To identify these blended absorption systems, we systematically cross-checked every line of the sub-DLA's D I Lyman series, to identify potential contamination with the Lyman series of an unrelated, higher redshift $\mathrm{H}$ I system. We also cross-checked each line of the sub-DLA's D I Lyman series against the expected positions of the metal-strong absorption line system located at $z_{\text {abs }}=2.053206$. In all cases, we used unblended lines of the contaminant system to determine more accurately the shape and depth of the absorption line that is blended with the sub-DLA. We have identified three D I Lyman series lines that are affected by blending; these include Ly $\epsilon$, Ly7, and Ly9, which we now discuss in turn.

In Figure 8, we show the D I Ly9 blend, which is due to Fe II $\lambda 1063 \AA$ absorption from an unrelated absorption complex at $z_{\mathrm{abs}}=2.053206$. The Fe II absorption is well modeled by the Fe II $\lambda 1608 \AA$ absorption, which falls in a clean part of the spectrum outside of the $\operatorname{Ly} \alpha$ forest and has roughly the same strength as the Fe II $\lambda 1063 \AA$ absorption.

The D I Ly $\epsilon$ blend is shown in Figure 9, and comprises two components at $z_{\mathrm{abs}}=2.39886 \pm 0.00005$ and $z_{\mathrm{abs}}=$ $2.39869 \pm 0.00013$, with $\mathrm{H} \mathrm{I}$ column densities of $\log _{10} N(\mathrm{H} \mathrm{I}) / \mathrm{cm}^{-2}=14.22 \pm 0.43$ and $\log _{10} N(\mathrm{H} \mathrm{I}) / \mathrm{cm}^{-2}=$ $14.18 \pm 0.48$, respectively. The total Doppler parameters of these absorption lines are $b_{\text {tot }}=21.4 \pm 1.5 \mathrm{~km} \mathrm{~s}^{-1}$ and $b_{\text {tot }}=$ $24.1 \pm 3.4 \mathrm{~km} \mathrm{~s}^{-1}$.

Finally, we find that the DI Ly7 line is blended with two separate (weak) absorption systems, which we present in Figure 10. The first blend is from the $\operatorname{Ly} \delta$ transition of a system at $z_{\text {abs }}=2.437351 \pm 0.000002$, which has an $\mathrm{HI}$ column density of $\log _{10} N(\mathrm{HI}) / \mathrm{cm}^{-2}=13.594 \pm 0.005$ and a Doppler parameter $b_{\text {tot }}=23.5 \pm 0.3 \mathrm{~km} \mathrm{~s}^{-1}$. The second blend is due to the $\operatorname{Ly} \beta$ absorption from a system at $z_{\text {abs }}=2.182786 \pm$ 


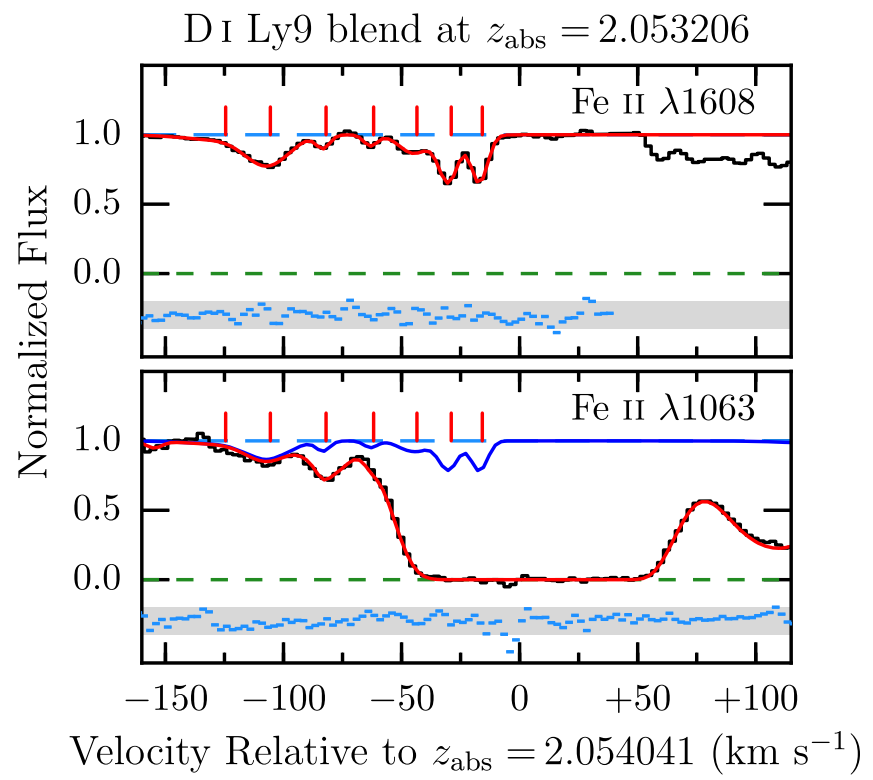

Figure 8. D I Ly9 of the sub-DLA (bottom panel) is blended with an Fe II $\lambda 1063$ complex at $z_{\text {abs }}=2.053206$. The top panel shows the corresponding Fe II $\lambda 1608$ absorption line of the contaminant system. In both panels, the red line shows the best model fit to the data (black histogram). The blue curve in the bottom panel shows the contribution of the blend to the total absorption profile. The long blue dashed lines represent the continuum levels, while the short green dashed line indicates the zero levels. The red tick marks above each spectrum indicate the absorption components of the blend. The blue points below each spectrum are the normalized fit residuals, (data-model)/error, of all pixels used in the analysis, and the gray band represents a confidence interval of $\pm 2 \sigma$.

0.000006. The H I column density and Doppler parameter of this system are $\log _{10} N(\mathrm{HI}) / \mathrm{cm}^{-2}=13.15 \pm 0.02$ and $b_{\text {tot }}=$ $34.5 \pm 1.1 \mathrm{~km} \mathrm{~s}^{-1}$, respectively.

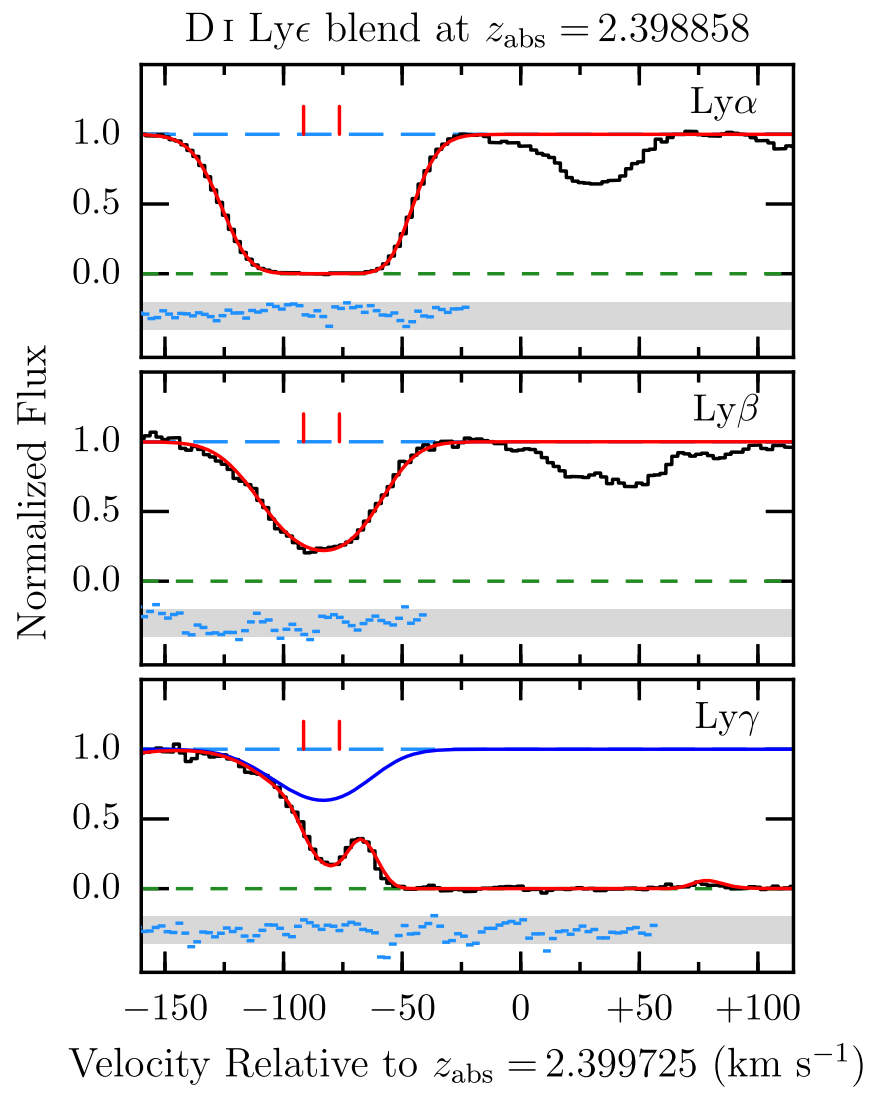

Figure 9. Same as Figure 8, but illustrating the blended D I Ly $\epsilon$ transition of the sub-DLA (bottom panel), due to an H I Ly $\gamma$ absorption line of an unrelated system at $z_{\text {abs }}=2.3988$ (see the blue profile in the bottom panel). The corresponding $\operatorname{Ly} \alpha$ and $\operatorname{Ly} \beta$ lines of this blend are shown in the top and middle panel, respectively. 


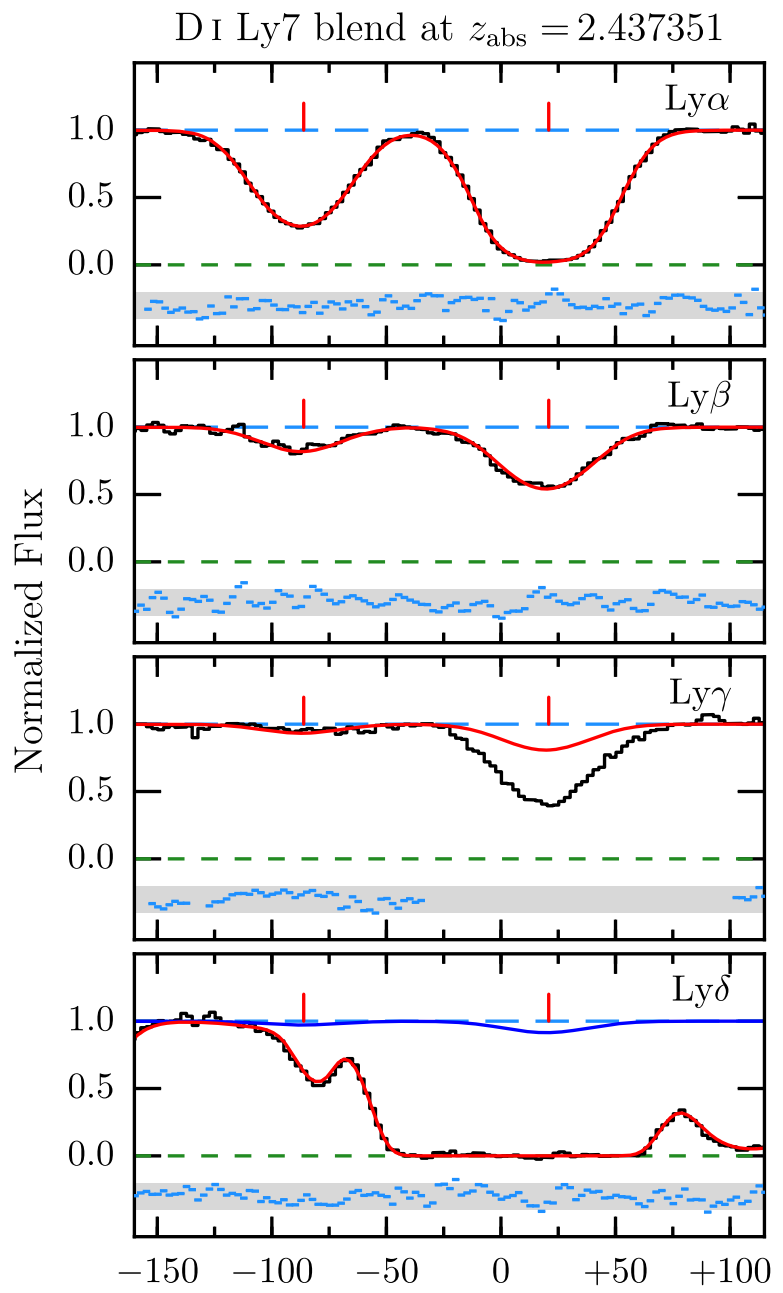

Velocity Relative to $z_{\mathrm{abs}}=2.438339\left(\mathrm{~km} \mathrm{~s}^{-1}\right)$

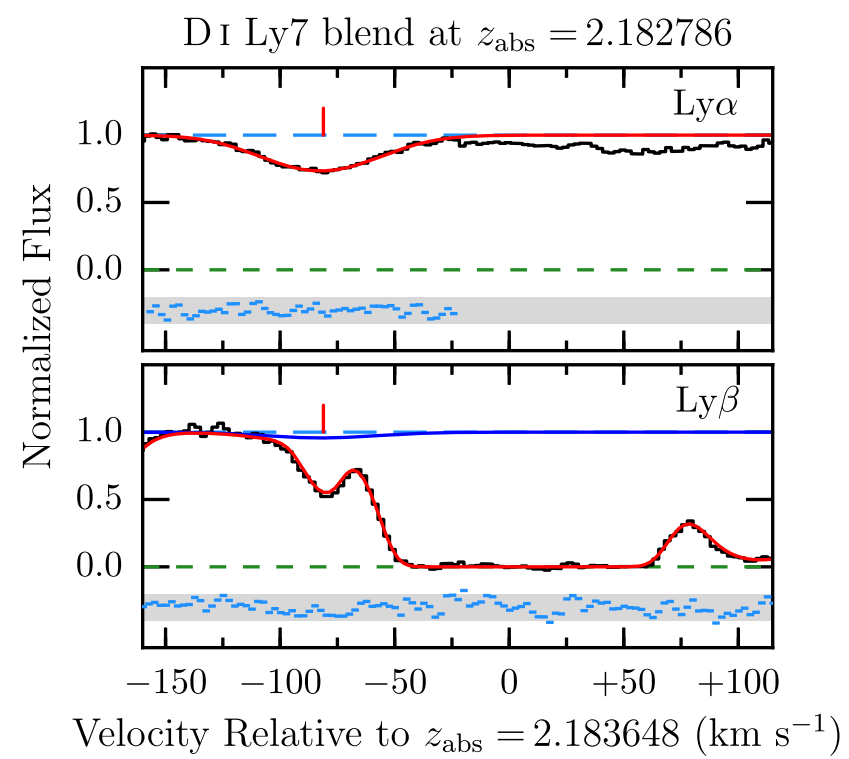

Figure 10. Same as Figures 8 and 9, but illustrating the two blends associated with the D I Ly7 transition of the sub-DLA.

\section{ORCID iDs}

Ryan J. Cooke (iD https://orcid.org/0000-0001-7653-5827

Charles C. Steidel (iD https://orcid.org/0000-0002-4834-7260

\section{References}

Adams, T. F. 1976, A\&A, 50, 461

Adelberger, E. G., García, A., Robertson, R. G. H., et al. 2011, RvMP, 83, 195

Akerman, C. J., Ellison, S. L., Pettini, M., \& Steidel, C. C. 2005, A\&A, 440, 499

Aoki, W., Barklem, P. S., Beers, T. C., et al. 2009, ApJ, 698, 1803

Asplund, M., Grevesse, N., Sauval, A. J., \& Scott, P. 2009, ARA\&A, 47, 481

Asplund, M., Lambert, D. L., Nissen, P. E., Primas, F., \& Smith, V. V. 2006, ApJ, 644, 229

Astropy Collaboration et al. 2013, A\&A, 558, A33

Aver, E., Olive, K. A., \& Skillman, E. D. 2015, JCAP, 7, 011

Balashev, S. A., Zavarygin, E. O., Ivanchik, A. V., Telikova, K. N., \& Varshalovich, D. A. 2016, MNRAS, 458, 2188

Bania, T. M., Rood, R. T., \& Balser, D. S. 2002, Natur, 415, 54

Bernstein, R. M., Burles, S. M., \& Prochaska, J. X. 2015, PASP, 127, 911

Burles, S., \& Tytler, D. 1998a, ApJ, 499, 699

Burles, S., \& Tytler, D. 1998b, ApJ, 507, 732

Cooke, R. 2015, ApJ, 812, L12

Cooke, R., \& Pettini, M. 2016, MNRAS, 455, 1512

Cooke, R. J., Pettini, M., \& Jorgenson, R. A. 2015, ApJ, 800, 12

Cooke, R. J., Pettini, M., Jorgenson, R. A., Murphy, M. T., \& Steidel, C. C. 2014, ApJ, 781, 31
Cooke, R. J., Pettini, M., Nollett, K. M., \& Jorgenson, R. 2016, ApJ, 830 , 148

Crighton, N. H. M., Webb, J. K., Ortiz-Gil, A., \& Fernández-Soto, A. 2004, MNRAS, 355, 1042

Cyburt, R. H., Fields, B. D., Olive, K. A., \& Yeh, T.-H. 2016, RvMP, 88 015004

Draine, B. T. 2004, in Proc. Origin and Evolution of the Elements Symp., ed. A. McWilliams \& M. Rauch (Cambridge: Cambridge Univ. Press), 317

Draine, B. T. 2006, in ASP Conf. Ser. 348, Astrophysics in the Far Ultraviolet: Five Years of Discovery with FUSE, ed. G. Sonneborn, H. Moos, \& B.-G. Andersson (San Francisco, CA: ASP), 58

Dvorkin, I., Vangioni, E., Silk, J., Petitjean, P., \& Olive, K. A. 2016, MNRAS, 458, L104

Ellison, S. L., Prochaska, J. X., \& Lopez, S. 2007, MNRAS, 380, 1245

Field, G. B., \& Steigman, G. 1971, ApJ, 166, 59

Fumagalli, M., O’Meara, J. M., \& Prochaska, J. X. 2011, Sci, 334, 1245

Grohs, E., Fuller, G. M., Kishimoto, C. T., Paris, M. W., \& Vlasenko, A. 2015 , PhRvD, 93, 083522

Gustavino, C. 2014, Proc. 52nd Int. Winter Meeting on Nuclear Physics (Trieste: POS), 050

Hunter, J. D. 2007, CSE, 9, 90

Izotov, Y. I., Thuan, T. X., \& Guseva, N. G. 2014, MNRAS, 445, 778

Jorgenson, R. A., Murphy, M. T., \& Thompson, R. 2013, MNRAS, 435, 482 Jura, M. 1982, in NASA CP 2238, Advances in UV Astronomy: 4 Years of IUE Research, ed. Y. Kondo, J. M. Mead, \& R. D. Chapman (Greenbelt, MD: NASA), 54

Kelson, D. D. 2003, PASP, 115, 688

Kirkman, D., Tytler, D., Suzuki, N., O'Meara, J. M., \& Lubin, D. 2003, ApJS, 149,1

Kochanek, I. 2016, J. Phys. Conf. Ser., 703, 012023 
Lallement, R., Hébrard, G., \& Welsh, B. Y. 2008, A\&A, 481, 381

Ledoux, C., Petitjean, P., Fynbo, J. P. U., Møller, P., \& Srianand, R. 2006, A\&A, 457, 71

Lehner, N., O’Meara, J. M., Fox, A. J., et al. 2014, ApJ, 788, 119

Linsky, J. L., Draine, B. T., Moos, H. W., et al. 2006, ApJ, 647, 1106

Mangano, G., Miele, G., Pastor, S., et al. 2005, NPhB, 729, 221

Marcucci, L. E., Mangano, G., Kievsky, A., \& Viviani, M. 2016, PhRvL, 116, 102501

Mathews, G. J., Kusakabe, M., \& Kajino, T. 2017, IJMPE, 26, 1741001

McQuinn, M., \& Switzer, E. R. 2009, PhRvD, 80, 063010

Meléndez, J., Casagrande, L., Ramírez, I., Asplund, M., \& Schuster, W. J. 2010, A\&A, 515, L3

Murphy, M. T., \& Bernet, M. L. 2016, MNRAS, 455, 1043

Murphy, M. T., Curran, S. J., Webb, J. K., Ménager, H., \& Zych, B. J. 2007, MNRAS, 376, 673

Neeleman, M., Wolfe, A. M., Prochaska, J. X., \& Rafelski, M. 2013, ApJ, 769, 54

Nollett, K. M., \& Burles, S. 2000, PhRvD, 61, 123505

Nollett, K. M., \& Holder, G. P. 2011, arXiv:1112.2683

Noterdaeme, P., López, S., Dumont, V., et al. 2012, A\&A, 542, L33

O'Meara, J. M., Burles, S., Prochaska, J. X., et al. 2006, ApJ, 649, L61

O'Meara, J. M., Lehner, N., Howk, J. C., et al. 2017, AJ, 154, 114

O’Meara, J. M., Lehner, N., Howk, J. C., et al. 2015, AJ, 150, 111

O’Meara, J. M., Tytler, D., Kirkman, D., et al. 2001, ApJ, 552, 718

Pettini, M., \& Bowen, D. V. 2001, ApJ, 560, 41

Pettini, M., \& Cooke, R. 2012, MNRAS, 425, 2477

Pettini, M., King, D. L., Smith, L. J., \& Hunstead, R. W. 1997, ApJ, 478, 536
Pettini, M., Zych, B. J., Murphy, M. T., Lewis, A., \& Steidel, C. C. 2008, MNRAS, 391, 1499

Planck Collaboration, Ade, P. A. R., Aghanim, N., et al. 2016, A\&A, 594, A13

Prochaska, J. X., Chen, H.-W., Wolfe, A. M., Dessauges-Zavadsky, M., \& Bloom, J. S. 2008, ApJ, 672, 59

Prochaska, J. X., Tripp, T. M., \& Howk, J. C. 2005, ApJ, 620, L39

Prodanović, T., Steigman, G., \& Fields, B. D. 2010, MNRAS, 406, 1108

Riemer-Sørensen, S., Kotuš, S., Webb, J. K., et al. 2017, MNRAS, 468, 3239

Riemer-Sørensen, S., Webb, J. K., Crighton, N., et al. 2015, MNRAS, 447, 2925

Savin, D. W. 2002, ApJ, 566, 599

Sbordone, L., Bonifacio, P., Caffau, E., et al. 2010, A\&A, 522, A26

Spite, M., Spite, F., Caffau, E., \& Bonifacio, P. 2015, A\&A, 582, A74

Steigman, G. 2001, in Proc. STScI Symp. Ser. 15, The Dark Universe: Matter, Energy and Gravity, ed. M. Livio (Cambridge: Cambridge Univ. Press), 46 Steigman, G. 2006, JCAP, 10, 016

Steigman, G. 2007, ARNPS, 57, 463

Suzuki, N., Tytler, D., Kirkman, D., O’Meara, J. M., \& Lubin, D. 2003, PASP, 115,1050

van de Voort, F., Quataert, E., Faucher-Giguère, C.-A., et al. 2017, MNRAS, submitted (arXiv:1704.08254)

van der Walt, S., Colbert, S. C., \& Varoquaux, G. 2011, CSE, 13, 22

Vladilo, G. 2004, A\&A, 421, 479

Vogt, S. S., Allen, S. L., Bigelow, B. C., et al. 1994, Proc. SPIE, 2198, 362

Whitmore, J. B., \& Murphy, M. T. 2015, MNRAS, 447, 446

Wood, B. E., Linsky, J. L., Hébrard, G., et al. 2004, ApJ, 609, 838

Zavarygin, E. O., Webb, J. K., Dumont, V., \& Riemer-Sørensen, S. 2017, MNRAS, submitted (arXiv:1706.09512) 